\title{
GEOMETRIC CONSTRUCTION OF QUASICONFORMAL MAPPINGS IN THE HEISENBERG GROUP
}

\author{
ROBIN TIMSIT
}

\begin{abstract}
In this paper, we are interested in the construction of quasiconformal mappings between domains of the Heisenberg group $\mathbf{H}$ that minimize a mean distortion functional. We propose to construct such mappings by considering a corresponding problem between domains of Poincaré half-plane $\mathbb{H}$ and then, lifting every of its solutions to $\mathbf{H}$. The first map we construct is a quasiconformal map between two cylinders. We explain the method used to find it and prove its uniqueness up to rotations. Then, we give geometric conditions which ensure that a minimizer (in $\mathbf{H}$ ) comes as a lift of a minimizer between domains of $\mathbb{H}$. Finally, as a non-trivial example of the generalization, we manage to reconstruct the map from [Ann. Acad. Sci. Fenn. Math. 38 (2013), pp. 149-180] between two spherical annuli and prove its uniqueness as a minimizer.
\end{abstract}

\section{INTRODUCTION AND STATEMENT OF RESULTS}

The theory of quasiconformal mappings in the complex plane is known to be a powerful tool to study deformations of complex structures. In spherical CR geometry, an adapted theory of quasiconformal mappings has been developed KR85, KR95] and used to define a distance in an analogue of Teichmüller space [Wan06]. In the case of spherical CR geometry, extremal quasiconformal mappings are still to be understood. Recently, some progress has been made in the area. A method using modulus of curve families has been developed [BFP13] in order to understand when a quasiconformal map has minimal mean distortion. In particular, the authors gave a condition, once we have a candidate for minimizing a mean distortion, to verify if it is indeed a minimizer. Minimizing a mean distortion functional is different from the classical Grötzsch problem which concerns minimizers for maximal distortion. However, the modulus of curve families method can be used to find lower bounds for maximal distortion and so, it can also be used to minimize maximal distortion (see [Tan96] for this type of argument in CR geometry). Here, we are interested in a new method to construct candidates for minimizing a mean distortion functional. For other uses of modulus of curve families in CR geometry, we may quote Min94, Kim14 who studied quasiconformal conjugacy classes of CR-diffeomorphisms of the 3-dimensional sphere.

In order to state our results, let us set notation and recall preliminary facts about the theory of quasiconformal mappings in the Heisenberg group. First, the Heisenberg group $\mathbf{H}$ is the set $\mathbb{C} \times \mathbb{R}$ with the group law: if $(z, t),\left(z^{\prime}, t^{\prime}\right) \in \mathbb{C} \times \mathbb{R}$,

\footnotetext{
Received by the editors December 6, 2016, and, in revised form, May 29, 2017, and March 23, 2018.

2010 Mathematics Subject Classification. Primary 30L10, 30C75.
} 
then

$$
(z, t) *\left(z^{\prime}, t^{\prime}\right)=\left(z+z^{\prime}, t+t^{\prime}+2 \Im\left(z \bar{z}^{\prime}\right)\right) .
$$

On $\mathbf{H}$, we have two left-invariant (complex) vector fields

$$
Z=\frac{\partial}{\partial z}+i \bar{z} \frac{\partial}{\partial t} \quad \text { and } \quad \bar{Z}=\frac{\partial}{\partial \bar{z}}-i z \frac{\partial}{\partial t} .
$$

If we set $T=\frac{\partial}{\partial t}$, one may verify that

$$
i[Z, \bar{Z}]=2 T \text {. }
$$

The other commutator relations give zero. Denoting the distribution $\operatorname{span}(Z)$ by $V, V$ is a CR structure on $\mathbf{H}$. It is known that the one point compactification of the Heisenberg group with this CR structure is CR-diffeomorphic to the 3-dimensional sphere endowed with its standard CR structure. Thus, the Heisenberg group is a local model of spherical CR geometry. Recall that a spherical CR-manifold is a $(G, X)$-manifold for $G=P U(2,1)$ and $X$ the 3 -dimensional sphere.

A theory of quasiconformal mappings on the Heisenberg group was developped by Korányi and Reimann. In what follows, we recall a few facts about it. For details, refer to KR85, KR95]. The Heisenberg group is endowed with a left-invariant metric

$$
d_{\mathbf{H}}(p, q):=\left\|p^{-1} * q\right\|_{\mathbf{H}},
$$

where $\|(z, t)\|_{\mathbf{H}}:=\left(|z|^{4}+t^{2}\right)^{\frac{1}{4}}$ is the Heisenberg norm. By analogy with the classical case, a homeomorphism $f: \Omega \longrightarrow \Omega^{\prime}$ between domains of $\mathbf{H}$ is called quasiconformal if

$$
H(p, f):=\limsup _{r \rightarrow 0} \frac{\max _{\mathbf{H}(p, q)=r} d_{\mathbf{H}}(f(p), f(q))}{\min _{d_{\mathbf{H}}(p, q)=r} d_{\mathbf{H}}(f(p), f(q))}, p \in \Omega
$$

is uniformly bounded. We say that $f$ is $K$-quasiconformal if $\|H(., f)\|_{L^{\infty}} \leq K$. As in the case of the complex plane, we have equivalent analytic definitions of quasiconformality. A sufficiently regular ( $C^{2}$ is enough) quasiconformal map between domains of $\mathbf{H}$ has to be a contact map for the contact structure induced by the form $\omega=\mathrm{d} t-i \bar{z} \mathrm{~d} z+i z \mathrm{~d} \bar{z}$, meaning that $f^{*} \omega=\lambda \omega$ for a nowhere vanishing real function $\lambda$. Moreover, denoting $f=\left(f_{1}, f_{2}\right)$ with $f_{1}$ the complex part of the map and $f_{2}$ the real one, then, if $f$ is an orientation-preserving quasiconformal map, it satisfies a system of PDEs quite similar to a Beltrami equation. Indeed, in that case, there is a complex valued function $\mu \in L^{\infty}$ (called a Beltrami coefficient) with $\|\mu\|_{L^{\infty}}<1$ such that

$$
\bar{Z} f_{1}=\mu Z f_{1} \text { and } \bar{Z}\left(f_{2}+i\left|f_{1}\right|^{2}\right)=\mu Z\left(f_{2}+i\left|f_{1}\right|^{2}\right) \text { a.e. }
$$

We then define the distortion function of the map $f$ by

$$
K(p, f):=\frac{1+|\mu(p)|}{1-|\mu(p)|}=\frac{\left|Z f_{1}(p)\right|+\left|\bar{Z} f_{1}(p)\right|}{\left|Z f_{1}(p)\right|-\left|\bar{Z} f_{1}(p)\right|}
$$

for $p \in \Omega$ where it makes sense and the maximal distortion of $f$ by

$$
K_{f}:=\underset{p \in \Omega}{\operatorname{ess} \sup } K(p, f) .
$$

It is known that a conformal (i.e., 1-quasiconformal) map $f: \Omega \longrightarrow \Omega^{\prime}$ is the restriction to $\Omega$ of the action of an element of $S U(2,1)$ (see [KR85, p. 337] for the smooth case and Cap97, p. 869] for the general one). 
Here, we are interested in the following minimization problem: consider a set of quasiconformal mappings $\mathcal{F} \subset\left\{f: \Omega \longrightarrow \Omega^{\prime}\right.$ q.c. $\}$. We are looking for a quasiconformal map $f_{0} \in \mathcal{F}$ such that

$$
\int_{\Omega} K\left(p, f_{0}\right)^{2} \rho_{0}^{4}(p) \mathrm{d} L^{3}(p)=\min _{f \in \mathcal{F}} \int_{\Omega} K(p, f)^{2} \rho_{0}^{4}(p) \mathrm{d} L^{3}(p)
$$

for a density $\rho_{0}$ depending on the geometry of the domain $\Omega$ and where $\mathrm{d} L^{3}$ is the Lebesgue measure on $\mathbb{R}^{3}$. If $f_{0} \in \mathcal{F}$ satisfies (1), we say that $f_{0}$ minimizes the mean distortion on $\mathcal{F}$ for the density $\rho_{0}$.

We propose here a geometric way to construct such minimizers in specific cases. The construction relies on the projection

$$
\begin{array}{ccc}
\Pi: \quad \mathbf{H} \backslash(\{0\} \times \mathbb{R}) & \longrightarrow & \mathbb{H} \\
(z, t) & \longmapsto t+i|z|^{2} .
\end{array}
$$

This projection comes from the CR identification between the Heisenberg group and the boundary of Siegel domain $E=\left\{\left.(z, w) \in \mathbb{C}^{2}|\Im(w)>| z\right|^{2}\right\}$ (that itself comes from the identification of standard CR structures of the one-point compactification of the Heisenberg group and the 3-dimensional sphere). The boundary of Siegel domain is identified with $\mathbb{C} \times \mathbb{R}$ by $(z, w) \longmapsto(z, \Re(w))$. Moreover, $\partial E \backslash\{z=$ $0\}$ is also diffeomorphic to a trivial circle bundle over the upper half-plane $\mathbb{H}$ by $(z, w) \longmapsto\left(\frac{z}{\sqrt{\Im(w)}}, w\right)$. So, identifying $\partial E \backslash\{z=0\}$ with $\mathbf{H} \backslash(\{0\} \times \mathbb{R})$, it gives a diffeomorphism

$$
\begin{aligned}
& \Psi^{-1}: \quad \mathbf{H} \backslash(\{0\} \times \mathbb{R}) \quad \longrightarrow \quad S^{1} \times \mathbb{H} \\
& (z, t) \quad \longmapsto\left(\frac{z}{|z|}, t+i|z|^{2}\right)
\end{aligned}
$$

and the projection $\Pi$, is simply the second component of that diffeomorphism. The (topological) identification of $\mathbf{H} \backslash\{z=0\}$ with a trivial circle bundle over the hyperbolic plane isn't new. For example, Korányi and Reimann in KR87. introduced coordinates

$$
(a, \alpha, \varphi) \longmapsto\left(a \cos ^{\frac{1}{2}}(\alpha) e^{i \varphi}, a^{2} \sin (\alpha)\right)
$$

so that (up to rotation and conjugation), $\left(a^{2}, \alpha\right)$ correspond to polar coordinates in $\mathbb{H}$.

Throughout the paper, we will study the following two problems:

(1) When can a minimizer of a mean distortion between domains of $\mathbb{H}$ be lifted by $\Pi$ into a quasiconformal map between domains of $\mathbf{H}$ ?

(2) When does a minimizer of a mean distortion in $\mathbf{H}$ necessarily come as a lift of a quasiconformal map between domains of $\mathbb{H}$ ?

The idea is the following. Under appropriate geometric conditions on domains $\Omega$ and $\Omega^{\prime}$ and on the density $\rho_{0}$, we can define a corresponding minimization problem between two domains $U$ and $V$ of Poincaré half-plane. If we have a solution to the problem on $\mathbb{H}, g: U \longrightarrow V$ such that there is a quasiconformal map $f=\left(f_{1}, f_{2}\right)$ : $\Omega \longrightarrow \Omega^{\prime}$ verifying $\left(f_{2}+i\left|f_{1}\right|^{2}\right)(z, t)=g\left(t+i|z|^{2}\right)$, then $f$ will be a solution to the problem on the Heisenberg group (Proposition 1.12 and Corollary 1.13). We study more precisely an example between two cylinders. In that case, we manage to construct explicitly a unique (up to rotations) solution of the minimization problem on $\mathbf{H}$ by lifting every solution of the corresponding problem on the half-plane, leading to Propositions 2.3 and 2.4. Proposition 2.3 states that, in the case of the 
cylinder, there is only one solution of the corresponding problem in $\mathbb{H}$ that can be lifted by $\Pi$ into a quasiconformal map between cylinders and Proposition 2.4 ensures that those lifts minimize a mean distortion functional. Then, Theorem 2.5 states that a minimizer of the mean distortion functional considered between cylinders is inevitably the lift of a minimizer of the corresponding problem in $\mathbb{H}$. After that, we generalize the result obtained between cylinders to some domains of the Heisenberg group. Namely, under appropriate conditions on $\Omega, \Omega^{\prime}$ and the density $\rho_{0}$, a minimizer $f$ has to be a lift by $\Pi$ of a minimizer for the corresponding problem in $\mathbb{H}$ (Theorem 3.18). It reduces the problem of finding such a minimizer, to the resolution of an ordinary differential equation with boundary conditions (Proposition 3.7).

We suppose, in the whole paper, that every quasiconformal map considered is $C^{2}$ and orientation-preserving and every curve is $C^{1}$, which gives a more restrictive class of maps than what was considered in, e.g., BFP15.

The paper is organized as follow. In Section 1, we present some theoretical background about moduli of curve families and state the problem we consider in the Heisenberg group and its corresponding one in the half-plane. Section 2 deals with construction and uniqueness (up to rotations) of a minimizer of a mean distortion functional between cylinders. We then generalize the construction in Section 3 and explain when it is the only way to find such minimizers; as an application, we reconstruct the extremal quasiconformal map between two spherical annuli found in [BFP13] and prove its uniqueness as a minimizer of a mean distortion functional.

\section{Minimization problem COnSidered in $\mathbf{H}$ AND ITS CORRESPONDING ONE IN $\mathbb{H}$}

Modulus of a curve family. By analogy with the complex case, in order to understand extremal properties of a quasiconformal map between two domains of the Heisenberg group, we look at its behavior on a well chosen family of rectifiable curves (with respect to $d_{\mathbf{H}}$ ) that foliates the domain. We restrict the study here to $C^{1}$ curves and $C^{2}$ orientation-preserving quasiconformal mappings, but most of the results of this section were proved in a general case. First of all, rectifiable curves are horizontal.

Definition 1.1 (Horizontal curves). A $C^{1}$ curve $\left.\gamma:\right] a, b[\longrightarrow \mathbf{H}$ is called horizontal if its tangents are in the contact distribution $D=\operatorname{ker}(\omega)$. This condition is given explicitly by the following. Let $\left.\gamma(s)=\left(\gamma_{1}(s), \gamma_{2}(s)\right), s \in\right] a, b[$ be a curve in $\mathbf{H}$. Then, $\gamma$ is horizontal if and only if

$$
\left.\dot{\gamma}_{2}(s)=-2 \Im\left(\bar{\gamma}_{1}(s) \dot{\gamma}_{1}(s)\right) \text { for all } s \in\right] a, b[.
$$

We can then define the modulus of a family of horizontal curves.

Definition 1.2 (Modulus of a family of horizontal curves). Let $\Gamma$ be a family of horizontal curves in a domain $\Omega$ of $\mathbf{H}$. We denote $\operatorname{adm}(\Gamma)$ the set of measurable Borel functions $\rho: \Omega \longrightarrow[0,+\infty]$ such that $\int_{\gamma} \rho \mathrm{d} l:=\int_{a}^{b} \rho(\gamma(s))\left|\dot{\gamma}_{1}(s)\right| \mathrm{d} s \geq 1$ for all curves $\gamma \in \Gamma$. We call densities the elements of $\operatorname{adm}(\Gamma)$. The modulus of the family $\Gamma$ is then defined by

$$
M(\Gamma):=\inf _{\rho \in \operatorname{adm}(\Gamma)} \int_{\Omega} \rho(p)^{4} \mathrm{~d} L^{3}(p) .
$$

We say that a density $\rho_{0}$ is extremal if it verifies $M(\Gamma)=\int_{\Omega} \rho_{0}(p)^{4} \mathrm{~d} L^{3}(p)$. 
When an extremal density exists, it is essentially unique (Proposition 3.4 in [BFP15, p. 143]).

Lemma 1.3. If $\rho_{1}$ and $\rho_{2}$ are both an extremal density for a family $\Gamma$ of horizontal curves in a domain $\Omega$ of $\mathbf{H}$, then $\rho_{1}=\rho_{2}$ a.e. in $\Omega$.

There is a link between quasiconformality and modulus of a family of horizontal curves. Indeed, we have the following.

Proposition 1.4. Let $f: \Omega \longrightarrow \Omega^{\prime}$ be a quasiconformal map between domains of $\mathbf{H}$. Then, for every family of horizontal curves $\Gamma$ in $\Omega$ and every $\rho \in \operatorname{adm}(\Gamma)$, one has

$$
M(f(\Gamma)) \leq \int_{\Omega} K(p, f)^{2} \rho(p)^{4} \mathrm{~d} L^{3}(p)
$$

This result is a direct consequence of an essential notion we will need. Fixing a density $\rho$ and a $C^{1}$ quasiconformal map $f: \Omega \longrightarrow \Omega^{\prime}$, one may define a push-forward by $f$ of the density $\rho$ (see, e.g., BFP13]).

Definition/Proposition 1.5 (Push-forward density). Let $f=\left(f_{1}, f_{2}\right): \Omega \longrightarrow$ $\Omega^{\prime}$ be a quasiconformal mapping between two domains of $\mathbf{H}$, let $\Gamma$ be a family of horizontal curves in $\Omega$ and $\rho \in \operatorname{adm}(\Gamma)$. Then,

$$
\rho^{\prime}=\frac{\rho}{\left|Z f_{1}\right|-\left|\bar{Z} f_{1}\right|} \circ f^{-1} \in \operatorname{adm}(f(\Gamma)) .
$$

Moreover,

$$
\int_{\Omega^{\prime}} \rho^{\prime 4} \mathrm{~d} L^{3}=\int_{\Omega} K(., f)^{2} \rho^{4} \mathrm{~d} L^{3} .
$$

Proof. A simple application of the chain rule and the fact that every $\gamma \in \Gamma$ is horizontal lead to the following. For every curve $\left.\gamma=\left(\gamma_{1}, \gamma_{2}\right):\right] a, b[\longrightarrow \Omega, \gamma \in \Gamma$, one has

$$
\left.\left(f_{1} \circ \gamma\right)(s)=Z f_{1}(\gamma(s)) \dot{\gamma}_{1}(s)+\bar{Z} f_{1}(\gamma(s)) \dot{\bar{\gamma}}_{1}(s) \text { for every } s \in\right] a, b[.
$$

This leads to the important inequality: for every $s \in] a, b[$,

$$
\left|Z f_{1}(\gamma(s))\right|-\left|\bar{Z} f_{1}(\gamma(s))\right| \leq \frac{\left|\left(f_{1} \circ \gamma\right)(s)\right|}{\left|\dot{\gamma}_{1}(s)\right|} \leq\left|Z f_{1}(\gamma(s))\right|+\left|\bar{Z} f_{1}(\gamma(s))\right| .
$$

So, if $\gamma \in \Gamma$, using inequality (3) and the fact that $\rho \in \operatorname{adm}(\Gamma)$, we find

$$
\int_{f_{1} \circ \gamma} \rho^{\prime} \mathrm{d} l=\int_{a}^{b} \frac{\rho}{\left|Z f_{1}\right|-\left|\bar{Z} f_{1}\right|} \circ \gamma(s)\left|\left(f_{1} \circ \gamma\right)(s)\right| \mathrm{d} s \geq \int_{a}^{b} \rho(\gamma(s))\left|\dot{\gamma}_{1}(s)\right| \mathrm{d} s \geq 1 .
$$

For the second part, this is simply an application of the following change of variable formula for quasiconformal mappings (Theorem 16 in [BFP13, p. 175]). For every non-negative measurable function $u: \Omega^{\prime} \longrightarrow \mathbb{R}$, we have

$$
\int_{\Omega}(u \circ f)(p)|J(p, f)| \mathrm{d} L^{3}(p)=\int_{\Omega^{\prime}} u(q) \mathrm{d} L^{3}(q),
$$

where $J(p, f)=\left(\left|Z f_{1}(p)\right|^{2}-\left|\bar{Z} f_{1}(p)\right|^{2}\right)^{2}$. So, using this formula and the definition of $\rho^{\prime}$, we have

$$
\int_{\Omega^{\prime}} \rho^{\prime} \mathrm{d} L^{3}=\int_{\Omega} \rho^{4} \frac{\left(\left|Z f_{1}\right|^{2}-\left|\bar{Z} f_{1}\right|^{2}\right)^{2}}{\left(\left|Z f_{1}\right|-\left|\bar{Z} f_{1}\right|\right)^{4}} \mathrm{~d} L^{3}=\int_{\Omega} K(., f)^{2} \rho^{4} \mathrm{~d} L^{3} .
$$


Just applying the previous proves Proposition 1.4. Now, in order to find a minimizer of a mean distortion in a class of quasiconformal maps, a first place to look would be for maps realizing equality in inequality (2) and then, use the following (obvious) proposition.

Proposition 1.6. Let $\Omega, \Omega^{\prime}$ be domains of $\mathbf{H}$, let $\mathcal{F}$ be a subset of the set of all orientation-preserving quasiconformal maps from $\Omega$ to $\Omega^{\prime}$, and let $\Gamma_{0}$ be a family of horizontal curves in $\Omega$ with $\rho \in \operatorname{adm}\left(\Gamma_{0}\right)$. Suppose that $f_{0} \in \mathcal{F}$ satisfies

$$
M\left(f_{0}\left(\Gamma_{0}\right)\right)=\int_{\Omega} K\left(., f_{0}\right)^{2} \rho^{4} \mathrm{~d} L^{3}
$$

and that there is a larger family $\Gamma \supset \Gamma_{0}$ of horizontal curves in $\Omega$ with $\rho \in \operatorname{adm}(\Gamma)$ and verifying

$$
M\left(f_{0}\left(\Gamma_{0}\right)\right) \leq M(f(\Gamma))
$$

for every $f \in \mathcal{F}$. Then, $f_{0}$ minimizes the mean distortion in $\mathcal{F}$ for the density $\rho$.

Proof. Proposition 1.4 ensures that for every $f \in \mathcal{F}$ we have

$$
M(f(\Gamma)) \leq \int_{\Omega} K(., f)^{2} \rho^{4} \mathrm{~d} L^{3} .
$$

Moreover, for every $f \in \mathcal{F}$,

$$
\int_{\Omega} K\left(., f_{0}\right)^{2} \rho^{4} \mathrm{~d} L^{3}=M\left(f_{0}\left(\Gamma_{0}\right)\right) \leq M(f(\Gamma)) .
$$

So, for every $f \in \mathcal{F}$,

$$
\int_{\Omega} K\left(., f_{0}\right)^{2} \rho^{4} \mathrm{~d} L^{3} \leq \int_{\Omega} K(., f)^{2} \rho^{4} \mathrm{~d} L^{3} .
$$

Theorem 1 in [BFP13, p. 153] gives a sufficient condition on a map to ensure that it minimizes the mean distortion in a subset of quasiconformal maps between bounded domains of $\mathbf{H}$ for a nice density.

Theorem 1.7. Let $\Omega$ and $\Omega^{\prime}$ be bounded domains of $\mathbf{H}$. Let $\left.\gamma:\right] a, b[\times \Lambda \longrightarrow \Omega$ be a diffeomorphism that foliates $\Omega$, where $] a, b[\subset \mathbb{R}$ with $a>0$ and $\Lambda$ is a domain of $\mathbb{R}^{2}$, such that $\gamma(\cdot, \lambda)$ is a horizontal curve verifying $\left|\dot{\gamma}_{1}(s, \lambda)\right| \neq 0$ for all $\lambda \in \Lambda$ and $\mathrm{d} L^{3}(\gamma(s, \lambda))=\left|\dot{\gamma}_{1}(s, \lambda)\right|^{4} \mathrm{~d} s \mathrm{~d} \mu(\lambda)$ for a measure $\mathrm{d} \mu$ on $\Lambda$.

Then,

$$
\rho_{0}(p):=\frac{1}{(b-a)\left|\dot{\gamma}_{1}\left(\gamma^{-1}(p)\right)\right|}
$$

is an extremal density for the family $\Gamma_{0}:=\{\gamma(\cdot, \lambda) \mid \lambda \in \Lambda\}$.

Moreover, if $\mathcal{F}$ is a subset of the set of all quasiconformal maps from $\Omega$ on $\Omega^{\prime}$ and $f_{0} \in \mathcal{F}$ is such that:

1) $\mu_{f_{0}}(\gamma(s)) \frac{\dot{\bar{\gamma}}_{1}(s)}{\dot{\gamma}_{1}(s)}<0$ for all $\left.s \in\right] a, b[$,

2) for all $\lambda \in \Lambda, K\left(\gamma(s, \lambda), f_{0}\right)$ does not depend on $s$,

3) there is $\Gamma \supset \Gamma_{0}$ such that $\rho_{0} \in \operatorname{adm}(\Gamma)$ and $M\left(f_{0}\left(\Gamma_{0}\right)\right) \leq M(f(\Gamma))$ for all $f \in \mathcal{F}$.

Then $f_{0}$ minimizes the mean distortion in $\mathcal{F}$ for the extremal density $\rho_{0}$. 
Statement of the corresponding problem in $\mathbb{H}$. The previous theorem gives a way, once we have a candidate, to check if that candidate minimizes a mean distortion functional. But, finding such a candidate may be quite challenging. Here, we explain how to construct such mappings in specific cases. As said in the introduction, the construction relies on the identification of $\mathbf{H} \backslash\{z=0\}$ with $S^{1} \times \mathbb{H}$ where $S^{1}$ is the unit circle of $\mathbb{C}$ and $\mathbb{H}$ is the Poincaré half-plane, identification given by the diffeomorphism $\Psi^{-1}$ defined in the introduction whose inverse is the map

$$
\begin{aligned}
\Psi: \quad S^{1} \times \mathbb{H} & \longrightarrow \quad \mathbf{H} \backslash(\{0\} \times \mathbb{R}) \\
\left(e^{i \theta}, w\right) & \longmapsto\left(\sqrt{\Im(w)} e^{i \theta}, \Re(w)\right) .
\end{aligned}
$$

It gives new coordinates on $\mathbf{H} \backslash(\{0\} \times \mathbb{R})$ and a simple computation gives the following expression of vector fields $Z$ and $\bar{Z}$ :

$$
\begin{aligned}
& Z=2 i \sqrt{\Im(w)} e^{-i \theta} \partial_{w}-\frac{i e^{-i \theta}}{2 \sqrt{\Im(w)}} \partial_{\theta}, \\
& \bar{Z}=-2 i \sqrt{\Im(w)} e^{i \theta} \partial_{\bar{w}}+\frac{i e^{i \theta}}{2 \sqrt{\Im(w)}} \partial_{\theta},
\end{aligned}
$$

where $\partial_{w}=\frac{\partial}{\partial w}, \partial_{\bar{w}}=\frac{\partial}{\partial \bar{w}}$ and $\partial_{\theta}=\frac{\partial}{\partial \theta}$.

In the following, we consider $\widetilde{\Omega}$ and $\widetilde{\Omega}^{\prime}$ domains in $\mathbf{H} \backslash(\{0\} \times \mathbb{R})$ such that $\Psi^{-1}(\widetilde{\Omega})=S^{1} \times \Omega$ and $\Psi^{-1}\left(\widetilde{\Omega}^{\prime}\right)=S^{1} \times \Omega^{\prime}$ with $\Omega, \Omega^{\prime}$ domains of $\mathbb{H}$. We will look at lifts by $\Pi$ of curves in the half-plane.

Lemma 1.8. Let $\gamma:] a, b\left[\longrightarrow \mathbb{H}\right.$ be $a C^{1}$ curve. Then, the only horizontal curves on $\mathbf{H} \backslash(\{0\} \times \mathbb{R}), \widetilde{\gamma}=\left(\gamma_{1}, \gamma_{2}\right)$ such that $\Pi(\widetilde{\gamma})=\gamma$ are the curves $\left(\sqrt{\Im(\gamma)} e^{i \tau}, \Re(\gamma)\right)$ where $\dot{\tau}=-\frac{\Re(\dot{\gamma})}{2 \Im(\gamma)}$.

Proof. Saying that $\gamma_{2}+i\left|\gamma_{1}\right|^{2}=\gamma$ gives $\gamma_{2}=\Re(\gamma)$ and $\left|\gamma_{1}\right|=\sqrt{\Im(\gamma)}$. So, we only have to check that $\left(\sqrt{\Im(\gamma)} e^{i \tau}, \Re(\gamma)\right)$ is horizontal if and only if $\dot{\tau}=-\frac{\Re(\dot{\gamma})}{2 \Im(\gamma)}$, which is a simple application of the definition of a horizontal curve.

Remark 1.9. Notice that lifting a curve gives a one-parameter family of curves in $\mathbf{H}$, which are obtained by rotating one lift around the vertical axis. Moreover, for a family of curves in $\mathbb{H}, \Gamma$, we define

$$
\widetilde{\Gamma}=\{\widetilde{\gamma} \mid \Pi(\widetilde{\gamma}) \in \Gamma\}
$$

and call $\widetilde{\Gamma}$ the lifted family of $\Gamma$.

Before going further, let's recall how the modulus of a curve family is defined in $\mathbb{C}$. Let $\Gamma$ be a family of curves $\gamma:] a, b[\longrightarrow \Omega$ in a domain $\Omega$ of $\mathbb{C}$. We denote again $\operatorname{adm}(\Gamma)$ the set of measurable Borel functions $\rho: \Omega \longrightarrow[0, \infty]$ such that $\int_{\gamma} \rho \mathrm{d} l=\int_{a}^{b} \rho(\gamma(s))|\dot{\gamma}(s)| \mathrm{d} s \geq 1$. The modulus of the family $\Gamma$ is

$$
M(\Gamma)=\inf _{\rho \in \operatorname{adm}(\Gamma)} \int_{\Omega} \rho^{2} \mathrm{~d} L^{2},
$$

where $\mathrm{d} L^{2}$ is the Lebesgue measure of $\mathbb{R}^{2}$. With that in mind, we can define the pull-back by $\Pi$ of a density. 
Definition/Proposition 1.10 (Pull-back density). Let $\Omega$ be a domain in $\mathbb{H}$ and $\widetilde{\Omega}=\Psi\left(S^{1} \times \Omega\right)$. Let $\Gamma$ be a curve family in $\Omega$ and denote $\widetilde{\Gamma}$ its lifted family in $\widetilde{\Omega}$. If $\rho \in \operatorname{adm}(\Gamma)$, define $\widetilde{\rho}$ for every $(z, t) \in \widetilde{\Omega}$ by

$$
\widetilde{\rho}(z, t):=|Z \Pi(z, t)| \rho(\Pi(z, t))=2|z| \rho\left(t+i|z|^{2}\right) .
$$

Then $\widetilde{\rho} \in \operatorname{adm}(\widetilde{\Gamma})$. We call the density $\widetilde{\rho}$ the pull-back by $\Pi$ of $\rho$.

Proof. Let $\widetilde{\gamma}=\left(\gamma_{1}, \gamma_{2}\right) \in \widetilde{\Gamma}$. By definition, there is $\gamma \in \Gamma$ such that $\gamma_{2}+i\left|\gamma_{1}\right|^{2}=\gamma$. Since $\widetilde{\gamma}$ is horizontal, $\dot{\gamma}=2 i \bar{\gamma}_{1} \dot{\gamma}_{1}$. Using the definition of $\widetilde{\rho}$, we find

$$
\int_{\widetilde{\gamma}} \widetilde{\rho} \mathrm{d} l=\int_{a}^{b} 2\left|\gamma_{1}(s)\right| \rho(\gamma(s))\left|\dot{\gamma}_{1}(s)\right| \mathrm{d} s=\int_{a}^{b} \rho(\gamma(s))|\dot{\gamma}(s)| \mathrm{d} s \geq 1 .
$$

Notation 1.11.

- Let $\Gamma$ be a curve family in a domain $\Omega$ of $\mathbb{H}$ and $\rho \in \operatorname{adm}(\Gamma)$. Then, $\Pi^{*} \rho$ refers to the pull-back by $\Pi$ of $\rho$. Moreover, one can define the same notion of push-forward density in $\mathbb{C}$ by setting $g_{*} \rho=\frac{\rho}{\left|\partial_{w} g\right|-\left|\partial_{\bar{w}} g\right|} \circ g^{-1}$ for any quasiconformal map $g: \Omega \longrightarrow \Omega^{\prime} \subset \mathbb{C}$. In that case, the push-forward density satisfies

$$
\int_{\Omega^{\prime}}\left(g_{*} \rho\right)^{2} \mathrm{~d} L^{2}=\int_{\Omega} \rho^{2} K(., g) \mathrm{d} L^{2}
$$

where $K(., g)$ is the quasiconformal distortion function of $g$.

- Let $\widetilde{\Gamma}$ be a family of horizontal curves in a domain $\widetilde{\Omega}$ of $\mathbf{H}, \widetilde{\rho} \in \operatorname{adm}(\widetilde{\Gamma})$, and let $f: \widetilde{\Omega} \longrightarrow \widetilde{\Omega}^{\prime} \subset \mathbf{H}$ be a quasiconformal map. Then, the push-forward by $f$ of $\widetilde{\rho}$ is denoted by $f_{*} \widetilde{\rho}$.

The following proposition and corollary explain the link between some minimization problems in the Heisenberg group and corresponding problems in the half-plane.

Proposition 1.12. Let $\Omega$ and $\Omega^{\prime}$ be domains of $\mathbb{H}$. Denote $\widetilde{\Omega}:=\Psi\left(S^{1} \times \Omega\right)$ and $\widetilde{\Omega}^{\prime}:=\Psi\left(S^{1} \times \Omega^{\prime}\right)$. Let $\Gamma$ be a curve family in $\Omega$ and let $\widetilde{\Gamma}$ be its lifted family in $\widetilde{\Omega}$. If $\rho \in \operatorname{adm}(\Gamma)$ and $g: \Omega \longrightarrow \Omega^{\prime}$ is a quasiconformal map such that there is a quasiconformal map $f: \widetilde{\Omega} \longrightarrow \widetilde{\Omega}^{\prime}$ with $\Pi \circ f=g \circ \Pi$, then

$$
\Pi^{*}\left(g_{*} \rho\right)=f_{*}\left(\Pi^{*} \rho\right) .
$$

Before going through the proof, we give a corollary of this.

Corollary 1.13. Let $g: \Omega \longrightarrow \Omega^{\prime}$ and $f: \widetilde{\Omega} \longrightarrow \widetilde{\Omega}^{\prime}$ be as in the previous proposition. Let $\Gamma$ be a curve family in $\Omega$ with continuous extremal density $\rho_{0}$ and $\Gamma^{\prime}:=g(\Gamma)$ with continuous extremal density $\rho_{0}^{\prime}$. Suppose the following:

1) $M(\widetilde{\Gamma})=\int_{\widetilde{\Omega}}\left(\Pi^{*} \rho_{0}\right)^{4} \mathrm{~d} L^{3}$ where $\widetilde{\Gamma}$ is the lifted family of $\Gamma$,

2) $M\left(\widetilde{\Gamma}^{\prime}\right)=\int_{\widetilde{\Omega}^{\prime}}\left(\Pi^{*} \rho_{0}^{\prime}\right)^{4} \mathrm{~d} L^{3}$ where $\widetilde{\Gamma}^{\prime}$ is the lifted family of $\Gamma^{\prime}$.

Then,

if and only if

$$
M(f(\widetilde{\Gamma}))=\int_{\widetilde{\Omega}}\left(\Pi^{*} \rho_{0}\right)^{4} K(., f)^{2} \mathrm{~d} L^{3}
$$

$$
M(g(\Gamma))=\int_{\Omega} K(., g) \rho_{0}^{2} \mathrm{~d} L^{2} .
$$


Proof of Proposition 1.12, By definition, $g_{*} \rho=\frac{\rho}{\left|\partial_{w} g\right|-\left|\partial_{\bar{w}} g\right|} \circ g^{-1}$ and so

$$
\Pi^{*}\left(g_{*} \rho\right)=|Z \Pi|\left(\frac{\rho}{\left|\partial_{w} g\right|-\left|\partial_{\bar{w}} g\right|} \circ g^{-1} \circ \Pi\right) \text {. }
$$

Moreover, since $f$ is contact, one has (see [KR85, p. 335])

$$
Z(\Pi \circ f)=Z\left(f_{2}+i\left|f_{1}\right|\right)=2 i \bar{f}_{1} Z f_{1} \text { and } \bar{Z}(\Pi \circ f)=\bar{Z}\left(f_{2}+i\left|f_{1}\right|\right)=2 i \bar{f}_{1} \bar{Z} f_{1} \text {. }
$$

Thus, since $\Pi$ is a CR-function, using the chain rule we have

$$
\begin{aligned}
\left|Z f_{1}\right|-\left|\bar{Z} f_{1}\right| & =\frac{1}{2\left|f_{1}\right|}(|Z(\Pi \circ f)|-|\bar{Z}(\Pi \circ f)|) \\
& =\frac{1}{2\left|f_{1}\right|}(|Z(g \circ \Pi)|-|\bar{Z}(g \circ \Pi)|) \\
& =\frac{|Z \Pi|}{2\left|f_{1}\right|}\left(\left(\left|\partial_{w} g\right|-\left|\partial_{\bar{w}} g\right|\right) \circ \Pi\right) .
\end{aligned}
$$

Now, computing $f_{*}\left(\Pi^{*} \rho\right)$, we find

$$
\begin{aligned}
f_{*}\left(\Pi^{*} \rho\right) & =\frac{|Z \Pi|(\rho \circ \Pi)}{\left|Z f_{1}\right|-\left|\bar{Z} f_{1}\right|} \circ f^{-1} \\
& =2\left|f_{1} \circ f^{-1}\right|\left(\frac{\rho}{\left|\partial_{w} g\right|-\left|\partial_{\bar{w}} g\right|} \circ \Pi \circ f^{-1}\right) \\
& =|Z \Pi|\left(\frac{\rho}{\left|\partial_{w} g\right|-\left|\partial_{\bar{w}} g\right|} \circ g^{-1} \circ \Pi\right)
\end{aligned}
$$

Proof of Corollary 1.13, Notice that $\Gamma^{\prime}=g(\Gamma)=g(\Pi(\widetilde{\Gamma}))=\Pi(f(\widetilde{\Gamma}))$ and $\widetilde{\Gamma^{\prime}}=$ $f(\widetilde{\Gamma})$ :

$$
\begin{aligned}
\widetilde{\delta} \in f(\widetilde{\Gamma}) & \Longleftrightarrow f^{-1}(\widetilde{\delta}) \in \widetilde{\Gamma} \\
& \Longleftrightarrow \Pi \circ f^{-1}(\widetilde{\delta}) \in \Gamma \\
& \Longleftrightarrow g^{-1} \circ \Pi(\widetilde{\delta}) \in \Gamma \\
& \Longleftrightarrow \Pi(\widetilde{\delta}) \in \Gamma^{\prime} \\
& \Longleftrightarrow \widetilde{\delta} \in \widetilde{\Gamma}^{\prime} .
\end{aligned}
$$

Now, assume first that $M\left(\Gamma^{\prime}\right)=\int_{\Omega} K(., g) \rho_{0}^{2} \mathrm{~d} L^{2}$. Then, $g_{*} \rho_{0}$ and $\rho_{0}^{\prime}$ are both extremal for $\Gamma^{\prime}$. By a result similar to Lemma 1.3 for the complex plane, and continuity of $g_{*} \rho_{0}$ and $\rho_{0}^{\prime}, g_{*} \rho_{0}=\rho_{0}^{\prime}$ in $\Omega^{\prime}$. So

$$
f_{*} \Pi^{*} \rho_{0}=\Pi^{*} g_{*} \rho_{0}=\Pi^{*} \rho_{0}^{\prime}
$$

in $\widetilde{\Omega}^{\prime}$. Thus, we have

$$
M(f(\widetilde{\Gamma}))=M\left(\widetilde{\Gamma}^{\prime}\right)=\int_{\widetilde{\Omega}^{\prime}}\left(f_{*} \Pi^{*} \rho_{0}\right)^{4} \mathrm{~d} L^{3}=\int_{\widetilde{\Omega}} K(., f)^{2}\left(\Pi^{*} \rho_{0}\right)^{4} \mathrm{~d} L^{3} .
$$

Conversely, assume that $M(f(\widetilde{\Gamma}))=\int_{\widetilde{\Omega}^{\prime}}\left(f_{*} \Pi^{*} \rho_{0}\right)^{4} \mathrm{~d} L^{3}$. Then, we have

$$
\int_{\widetilde{\Omega}^{\prime}}\left(\Pi^{*} \rho_{0}^{\prime}\right)^{4} \mathrm{~d} L^{3}=M\left(\widetilde{\Gamma}^{\prime}\right)=\int_{\widetilde{\Omega}^{\prime}}\left(\Pi^{*} g_{*} \rho_{0}\right)^{4} \mathrm{~d} L^{3} .
$$


By Lemma 1.3, and continuity of densities, we deduce that $\Pi^{*} g_{*} \rho_{0}=\Pi^{*} \rho_{0}^{\prime}$ in $\widetilde{\Omega}^{\prime}$. Using the formula given for pull-back densities, one easily finds that $g_{*} \rho_{0}=\rho_{0}^{\prime}$. This leads to

$$
M\left(\Gamma^{\prime}\right)=M(g(\Gamma))=\int_{\Omega} K(., g) \rho_{0}^{2} \mathrm{~d} L^{2} .
$$

Lemma 1.14. Let $g: \Omega \longrightarrow \Omega^{\prime}$ be a map between domains of $\mathbb{H}$. Denote $\widetilde{\Omega}=$ $\Psi\left(S^{1} \times \Omega\right)$ and $\widetilde{\Omega}^{\prime}=\Psi\left(S^{1} \times \Omega^{\prime}\right)$. Assume that there is a contact map $f: \widetilde{\Omega} \longrightarrow \widetilde{\Omega}^{\prime}$ such that $\Pi \circ f=g \circ \Pi$. Then, for every $(z, t)$ in $\widetilde{\Omega}$,

$$
K((z, t), f)=K(\Pi(z, t), g) .
$$

This means that $f$ is $K$-quasiconformal if and only if $g$ is $K$-quasiconformal.

Proof. Just using

$$
Z(\Pi \circ f)=2 i \bar{f}_{1} Z f_{1} \quad \text { and } \quad \bar{Z}(\Pi \circ f)=2 i \bar{f}_{1} \bar{Z} f_{1}
$$

together with the fact that $\Pi$ is $\mathrm{CR}$, leads to

$$
\frac{\left|Z f_{1}\right|-\left|\bar{Z} f_{1}\right|}{\left|Z f_{1}\right|+\left|\bar{Z} f_{1}\right|}=\frac{\left|\partial_{w} g\right|-\left|\partial_{\bar{w}} g\right|}{\left|\partial_{w} g\right|+\left|\partial_{\bar{w}} g\right|} \circ \Pi
$$

which is exactly the statement of the lemma.

So, if we are looking for a quasiconformal map on the Heisenberg group that minimizes a mean distortion for a nice density, a first step would be to look for solutions of the corresponding problem in $\mathbb{H}$ that can be lifted by $\Pi$ into contact transformations. This is what we will do in the next section for cylinders.

\section{Construction and uniqueness of} AN EXTREMAL QUASICONFORMALMAP BETWEEN CYLINDERS

2.1. Construction of the map. In this section, we construct explicitly a minimizer of a mean distortion functional in a class of quasiconformal maps between cylinders. Our objective is to prove Proposition 2.4.

As said, we are looking for a quasiconformal map between cylinders defined as a lift by $\Pi$ of a quasiconformal map between projections of cylinders. For $r, R>0$, we denote $C_{r, R}$ the cylinder

$$
C_{r, R}=\{(z, t) \in \mathbf{H}|0<t<r \&| z \mid<\sqrt{R}\} .
$$

Here we are interested in finding a quasiconformal map $f: C_{a, b} \longrightarrow C_{a^{\prime}, b^{\prime}}$ with $\frac{a b^{\prime}}{a^{\prime} b}>1$ that minimizes a mean distortion functional within the set $\mathcal{F}$ of all orientation-preserving quasiconformal mappings from $C_{a, b}$ to $C_{a^{\prime}, b^{\prime}}$ that extend homeomorphically to the boundary and map

- the $\operatorname{disc}\{(z, t) \in \mathbf{H}|| z \mid \leq \sqrt{b} \& t=0\}$ to the $\operatorname{disc}\{(z, t) \in \mathbf{H}|| z \mid \leq$ $\left.\sqrt{b^{\prime}} \& t=0\right\}$,

- the disc $\{(z, t) \in \mathbf{H}|| z \mid \leq \sqrt{b} \& t=a\}$ to the $\operatorname{disc}\{(z, t) \in \mathbf{H}|| z \mid \leq$ $\left.\sqrt{b^{\prime}} \& t=a^{\prime}\right\}$,

- and the cylinder $\{(z, t) \in \mathbf{H}|| z \mid=\sqrt{b} \& 0 \leq t \leq a\}$ to the cylinder $\{(z, t) \in \mathbf{H}|| z \mid=\sqrt{b} \& 0 \leq t \leq a\}$. 
Consider the rectangles

$$
R_{a, b}=\{w \in \mathbb{C} \mid 0<\Re(w)<a, 0<\Im(w)<b\}
$$

and

$$
R_{a^{\prime}, b^{\prime}}=\left\{w \in \mathbb{C} \mid 0<\Re(w)<a^{\prime}, 0<\Im(w)<b^{\prime}\right\} .
$$

Then $\Pi\left(C_{a, b} \backslash\{z=0\}\right)=R_{a, b}$ and $\Pi\left(C_{a^{\prime}, b^{\prime}} \backslash\{z=0\}\right)=R_{a^{\prime}, b^{\prime}}$. On the cylinder, $C_{a, b}$, there is a natural foliation by horizontal curves given by

$$
\widetilde{\Gamma}_{0}=\left\{\widetilde{\gamma}_{z}(s)=\left(z e^{-\frac{i s}{2|z|^{2}}}, s\right)|0<| z \mid<\sqrt{b} \& 0<s<a\right\}
$$

which are the horizontal lifts by $\Pi$ of curves

$$
\gamma_{y}(s)=s+i y \text { for } 0<y<b
$$

on $R_{a, b}$ (see Figure 1).

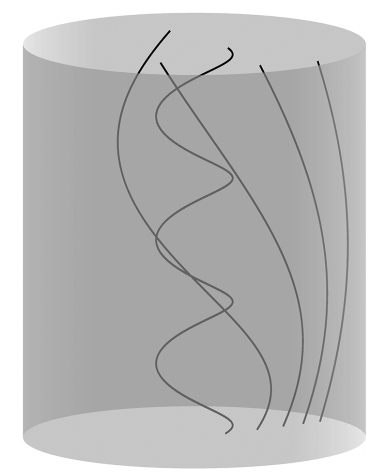

FIgure 1. Cylinder foliated by curves in $\widetilde{\Gamma}_{0}$ (foliation given by rotations around the vertical axis of drawn curves and the vertical axis itself).

To state the minimization problem we are dealing with, we need to find the modulus and extremal density of $\widetilde{\Gamma}_{0}$.

Lemma 2.1. The curve family $\widetilde{\Gamma}_{0}$ has modulus

$$
M\left(\widetilde{\Gamma}_{0}\right)=\frac{16 \pi b^{3}}{3 a^{3}}
$$

and its extremal density is $\widetilde{\rho}_{0}(z, t)=\frac{2|z|}{a}$.

Proof. If $\widetilde{\rho} \in \operatorname{adm}\left(\widetilde{\Gamma}_{0}\right)$, by definition we have

$$
\int_{\widetilde{\gamma}_{z}} \widetilde{\rho} \mathrm{d} l=\int_{0}^{a} \widetilde{\rho}\left(\widetilde{\gamma}_{z}(s)\right) \frac{\mathrm{d} s}{2|z|} \geq 1
$$


for all $0<|z|<\sqrt{b}$. So,

$$
\int_{0}^{a} \widetilde{\rho}\left(\widetilde{\gamma}_{z}(s)\right) \mathrm{d} s \geq 2|z|
$$

for all $0<|z|<\sqrt{b}$. But,

$$
\begin{array}{rlrl}
\int_{C_{a, b}} \widetilde{\rho}^{4} \mathrm{~d} L^{3} & & & = \\
\text { cylindrical coordinates } & & \int_{0}^{2 \pi} \int_{0}^{\sqrt{b}} \int_{0}^{a} \widetilde{\rho}^{4}(r, \theta, t) r \mathrm{~d} t \mathrm{~d} r \mathrm{~d} \theta \\
& = & \int_{0}^{2 \pi} \int_{0}^{\sqrt{b}} \int_{0}^{a} \widetilde{\rho}^{4}\left(r, \theta-\frac{s}{2 r^{2}}, s\right) r \mathrm{~d} s \mathrm{~d} r \mathrm{~d} \theta \\
\text { substitution } & = & & \int_{0}^{2 \pi} \int_{0}^{\sqrt{b}} \int_{0}^{a} \widetilde{\rho}^{4}\left(\widetilde{\gamma}_{r e^{i \theta}}(s)\right) r \mathrm{~d} s \mathrm{~d} r \mathrm{~d} \theta .
\end{array}
$$

Moreover,

$$
\begin{array}{rll}
\int_{0}^{a} \widetilde{\rho}^{4}\left(\widetilde{\gamma}_{r e^{i \theta}}(s)\right) \mathrm{d} s & \underset{\text { Hölder inequality }}{\geq} & \frac{1}{a^{3}}\left(\int_{0}^{a} \widetilde{\rho}\left(\widetilde{\gamma}_{r e^{i \theta}}(s)\right) \mathrm{d} s\right)^{4} \\
& \underset{\text { inequality (4) }}{\geq} & \frac{16 r^{4}}{a^{3}}
\end{array}
$$

for all $0<r<\sqrt{b}$ and $0<\theta<2 \pi$. Thus, we get

$$
\int_{C_{a, b}} \widetilde{\rho}^{4} \mathrm{~d} L^{3} \geq \frac{32 \pi}{a^{3}} \int_{0}^{\sqrt{b}} r^{5} \mathrm{~d} r=\frac{16 \pi b^{3}}{3 a^{3}}
$$

for all $\tilde{\rho} \in \operatorname{adm}\left(\widetilde{\Gamma}_{0}\right)$. Consequently,

$$
M\left(\widetilde{\Gamma}_{0}\right) \geq \frac{16 \pi b^{3}}{3 a^{3}} .
$$

Moreover, set

$$
\widetilde{\rho}_{0}(z, t)=\frac{2|z|}{a} .
$$

Then $\widetilde{\rho}_{0} \in \operatorname{adm}\left(\widetilde{\Gamma}_{0}\right)$ and

$$
\int_{C_{a, b}} \widetilde{\rho}_{0}^{4} \mathrm{~d} L^{3}=\frac{16 \pi b^{3}}{3 a^{3}}
$$

Let us set $\Gamma_{0}=\left\{\gamma_{y} \mid 0<y<b\right\}$ where $\left.\gamma_{y}:\right] 0, a\left[\longrightarrow R_{a, b}\right.$ is defined by $\gamma_{y}(s)=s+i y$. It is known since the work of Grötzsch that $\Gamma_{0}$ has modulus $\frac{b}{a}$ and extremal density $\rho_{0}(w)=\frac{1}{a}$. Let $\mathcal{G}$ be the set of all quasiconformal mappings from $R_{a, b}$ to $R_{a^{\prime}, b^{\prime}}$ that map homeomorphically the vertical and horizontal boundary components of $R_{a, b}$ on the corresponding ones of $R_{a^{\prime}, b^{\prime}}$. Here, and in the following, rectangles are considered as subsets of $\mathbb{C}$ and so, boundaries are considered in $\mathbb{C}$. The following is well known (a short proof is given in the appendix).

Lemma 2.2. Any minimizer of the mean distortion in $\mathcal{G}$ for the density $\rho_{0}$ is a map $f_{\varphi}(x+i y)=\frac{a^{\prime}}{a} x+i \varphi(y)$ where $\varphi:[0, b] \longrightarrow\left[0, b^{\prime}\right]$ is a diffeomorphism such that $\varphi(0)=0, \varphi(b)=b^{\prime}$, and $\dot{\varphi} \geq \frac{a^{\prime}}{a}$. 
Notice also that $\widetilde{\rho}_{0}=\Pi^{*} \rho_{0}$ and

$$
M\left(\widetilde{f_{\varphi}\left(\Gamma_{0}\right)}\right)=\int_{C_{a^{\prime}, b^{\prime}}}\left(\Pi^{*} \rho_{0}^{\prime}\right)^{4} \mathrm{~d} L^{3},
$$

where $\rho_{0}^{\prime}(w)=\frac{1}{a^{\prime}}$ is extremal for $f_{\varphi}\left(\Gamma_{0}\right)=\Gamma_{0}^{\prime}$ and $\Gamma_{0}^{\prime}$ is the family of horizontal lines in $R_{a^{\prime}, b^{\prime}}$ (which is true for every $\varphi$ defined as in the previous lemma). Moreover, every $f_{\varphi}$ defined as in the previous lemma satisfies

$$
M\left(f_{\varphi}\left(\Gamma_{0}\right)\right)=\int_{R_{a, b}} K\left(., f_{\varphi}\right) \rho_{0}^{2} \mathrm{~d} L^{2} .
$$

Then, according to Corollary 1.13 if we find a quasiconformal map $\tilde{f} \in \mathcal{F}$ that maps the vertical axis homeomorphically on the vertical axis and such that $\Pi \circ \tilde{f}=f_{\varphi} \circ \Pi$ for a function $\varphi$ defined as previously, then $\tilde{f}$ will satisfy

$$
M\left(\widetilde{f}\left(\widetilde{\Gamma}_{0}\right)\right)=\int_{C_{a, b}} K(., \widetilde{f})^{2} \widetilde{\rho}_{0}^{4} \mathrm{~d} L^{3} .
$$

Proposition 2.3. There is only one function $\varphi:[0, b] \longrightarrow\left[0, b^{\prime}\right]$ with $\varphi(0)=0$, $\varphi(b)=b^{\prime}$, and $\dot{\varphi} \geq \frac{a^{\prime}}{a}$ such that $f_{\varphi}$ can be lifted into a quasiconformal map $\tilde{f}$ : $C_{a, b} \longrightarrow C_{a^{\prime}, b^{\prime}}$. That function is defined for every $x \in[0, b]$ by

$$
\varphi(x)=\frac{b^{\prime} x}{\left(1-\frac{a b^{\prime}}{a^{\prime} b}\right) x+\frac{a b^{\prime}}{a^{\prime}}}
$$

and the lifts are compositions with rotations around the vertical axis of

$$
\begin{aligned}
& \widetilde{f}_{0}: C_{a, b} \longrightarrow C_{a^{\prime}, b^{\prime}} \\
& (z, t) \longmapsto\left(\frac{\sqrt{b^{\prime}} z e^{\frac{i}{2 b}\left(1-\frac{a^{\prime} b}{a b^{\prime}}\right) t}}{\sqrt{\left(1-\frac{a b^{\prime}}{a^{\prime} b}\right)|z|^{2}+\frac{a b^{\prime}}{a^{\prime}}}}, \frac{a^{\prime}}{a} t\right) .
\end{aligned}
$$

Proof. To prove this, it will be more convenient to compute in usual cylindrical coordinates on $\mathbf{H}=\mathbb{R}^{3}$, meaning $(r, \theta, t) \longmapsto\left(r e^{i \theta}, t\right)$. In those coordinates, the contact form writes as

$$
\omega=\mathrm{d} t+2 r^{2} \mathrm{~d} \theta .
$$

So, we are looking for four functions $R, \Theta, T, \varphi$ such that

$$
T+i R^{2}(r, \theta, t)=\frac{a^{\prime}}{a} t+i \varphi\left(r^{2}\right) \quad \text { and } \quad \mathrm{d} T+2 R^{2} \mathrm{~d} \Theta=\lambda\left(\mathrm{d} t+2 r^{2} \mathrm{~d} \theta\right)
$$

for a nowhere vanishing function $\lambda$. Moreover, for all $r, t, \Theta(r, ., t)$ is $2 \pi$-periodic modulo $2 \pi$. Since $T+i R^{2}(r, \theta, t)=\frac{a^{\prime}}{a} t+i \varphi\left(r^{2}\right)$, we get

$$
T(r, \theta, t)=T(t)=\frac{a^{\prime}}{a} t \quad \text { and } \quad R^{2}(r, \theta, t)=R^{2}(r)=\varphi\left(r^{2}\right) .
$$

The idea is to use the system of PDEs that the functions $R, \Theta$, and $T$ must verify in order to find an ordinary differential equation that $\varphi$ must verify. In the following, we denote by an index $r$ (resp., $\theta$, resp., $t$ ) the partial derivative of a function according to $r$ (resp., $\theta$, resp., $t$ ).

Since $T_{r}=T_{\theta}=0, T_{t}(t)=\frac{a^{\prime}}{a}$, and $\mathrm{d} T+2 R^{2} \mathrm{~d} \Theta=\lambda\left(\mathrm{d} t+2 r^{2} \mathrm{~d} \theta\right)$, we get that

$$
\Theta_{r}(r, \theta, t)=0 \quad \text { and } \quad \Theta_{\theta}(r, \theta, t)=\frac{a^{\prime} r^{2}}{a \varphi\left(r^{2}\right)}+2 r^{2} \Theta_{t}(r, \theta, t) \text {. }
$$


Moreover, from $\Theta_{r}=0$, we deduce that

$$
\Theta_{r, \theta}=\Theta_{r, t}=0 .
$$

So, differentiating $\Theta_{\theta}(r, \theta, t)=\frac{a^{\prime} r^{2}}{a \varphi\left(r^{2}\right)}+2 r^{2} \Theta_{t}(r, \theta, t)$ with respect to $r$ gives

$$
2 \Theta_{t}(r, \theta, t)=\frac{a^{\prime}}{a} \frac{r^{2} \dot{\varphi}\left(r^{2}\right)-\varphi\left(r^{2}\right)}{\varphi^{2}\left(r^{2}\right)}
$$

and we deduce

$$
\Theta_{\theta}(r, \theta, t)=\Theta_{\theta}(r)=\frac{a^{\prime} r^{4} \dot{\varphi}\left(r^{2}\right)}{a \varphi^{2}\left(r^{2}\right)} .
$$

From the fact that $\Theta_{r, \theta}=0$, putting $x=r^{2}, \varphi$ must verify the differential equation

$$
\frac{\mathrm{d}}{\mathrm{d} x}\left(\frac{x^{2} \dot{\varphi}(x)}{\varphi^{2}(x)}\right)=0
$$

whose solutions are the functions

$$
\varphi(x)=\frac{x}{C x+D} \text { with } C, D \in \mathbb{R} .
$$

For such functions, we have $\Theta_{\theta}(r, \theta, t)=\frac{a^{\prime}}{a} D$. So, for $(R, \Theta, T)$ to be a homeomorphism, we must have $D=\frac{a}{a^{\prime}}$. Moreover, we want that $\varphi(b)=b^{\prime}$, so $C=$ $\frac{1}{b^{\prime}}\left(1-\frac{a b^{\prime}}{a^{\prime} b}\right)$. Consequently,

$$
\varphi(x)=\frac{b^{\prime} x}{\left(1-\frac{a b^{\prime}}{a^{\prime} b}\right) x+\frac{a b^{\prime}}{a^{\prime}}}
$$

for all $x \in[0, b]$ (one may check that $\left(1-\frac{a b^{\prime}}{a^{\prime} b}\right) x+\frac{a b^{\prime}}{a^{\prime}}>0$ if $x \in[0, b]$ ). Moreover, it is easy to see that

$$
\dot{\varphi} \geq \frac{a^{\prime}}{a}
$$

Replacing $\varphi$ by its value, we find

$$
\begin{aligned}
& \Theta_{\theta}(r, \theta, t)=1, \\
& \Theta_{r}(r, \theta, t)=0, \\
& \Theta_{t}(r, \theta, t)=\frac{1}{2 b}-\frac{a^{\prime}}{2 a b^{\prime}} .
\end{aligned}
$$

And so

$$
\begin{aligned}
\Theta(r, \theta, t) & =\theta+\frac{1}{2}\left(\frac{1}{b}-\frac{a^{\prime}}{a b^{\prime}}\right) t+\alpha \text { with } \alpha \in \mathbb{R}, \\
R(r, \theta, t) & =\frac{\sqrt{b^{\prime}} r}{\sqrt{\left(1-\frac{a b^{\prime}}{a^{\prime} b}\right) r^{2}+\frac{a b^{\prime}}{a^{\prime}}}}, \\
T(r, \theta, t) & =\frac{a^{\prime}}{a} t,
\end{aligned}
$$

which gives in usual coordinates

$$
\widetilde{f}_{\alpha}(z, t)=\left(\frac{\sqrt{b^{\prime}} e^{i \alpha} z e^{\frac{i}{2 b}\left(1-\frac{a^{\prime} b}{a b^{\prime}}\right) t}}{\sqrt{\left(1-\frac{a b^{\prime}}{a^{\prime} b}\right)|z|^{2}+\frac{a b^{\prime}}{a^{\prime}}}}, \frac{a^{\prime}}{a} t\right) .
$$


The mappings $\widetilde{f}_{\alpha}$ are quasiconformal with distortion function

and maximal distortion

$$
K\left((z, t), \widetilde{f}_{\alpha}\right)=\frac{1}{\left(1+\left(\frac{a^{\prime}}{a b^{\prime}}-\frac{1}{b}\right)|z|^{2}\right)^{2}}
$$

$$
K_{\widetilde{f}_{\alpha}}=\left(\frac{a b^{\prime}}{a^{\prime} b}\right)^{2}
$$

Moreover, they map the vertical axis homeomorphically to the vertical axis, so according to what we said before the proposition, they satisfy

$$
M\left(\widetilde{f}_{\alpha}\left(\widetilde{\Gamma}_{0}\right)\right)=\int_{C_{a, b}} K\left(., \widetilde{f}_{\alpha}\right)^{4} \widetilde{\rho}_{0}^{4} \mathrm{~d} L^{3} .
$$

It remains to show that they minimize the mean distortion in $\mathcal{F}$ for the density $\widetilde{\rho}_{0}$. For that, we will use Proposition [1.6 and so, we need to find a larger family of horizontal curves $\widetilde{\Gamma} \supset \widetilde{\Gamma}_{0}$ for which $\widetilde{\rho}_{0} \in \operatorname{adm}(\widetilde{\Gamma})$ and $M\left(\widetilde{f}_{\alpha}\left(\widetilde{\Gamma}_{0}\right)\right) \leq M(f(\widetilde{\Gamma}))$ for every $f \in \mathcal{F}$.

Let $\widetilde{\Gamma}$ be the set of all horizontal curves in $C_{a, b}$ joining the discs $\overline{C_{a, b}} \cap\{t=0\}$ and $\overline{C_{a, b}} \cap\{t=a\}$. Then, we will see (first part of Lemma 2.6 ) that $\widetilde{\rho}_{0} \in \operatorname{adm}(\widetilde{\Gamma})$. Moreover, by definition of $\mathcal{F}$, for every $f \in \mathcal{F}, f(\widetilde{\Gamma})$ is the family of all horizontal curves in $C_{a^{\prime}, b^{\prime}}$ joining the two discs $\overline{C_{a^{\prime}, b^{\prime}}} \cap\{t=0\}$ and $\overline{C_{a^{\prime}, b^{\prime}}} \cap\left\{t=a^{\prime}\right\}$. So, for every $f \in \mathcal{F}, \widetilde{f}_{\alpha}\left(\widetilde{\Gamma}_{0}\right) \subset f(\widetilde{\Gamma})$ and thus we have $M\left(\widetilde{f}_{\alpha}\left(\widetilde{\Gamma}_{0}\right)\right) \leq M(f(\widetilde{\Gamma}))$ for every $f \in \mathcal{F}$. Applying Proposition [1.6 is enough to prove the following.

Proposition 2.4. Every composition by a rotation around the t-axis of the map

$$
\begin{aligned}
& \tilde{f}_{0}: C_{a, b} \quad \longrightarrow \quad C_{a^{\prime}, b^{\prime}} \\
& (z, t) \longmapsto\left(\frac{\sqrt{b^{\prime}} z e^{\frac{i}{2 b}\left(1-\frac{a^{\prime} b}{a b^{\prime}}\right) t}}{\sqrt{\left(1-\frac{a b^{\prime}}{a^{\prime} b}\right)|z|^{2}+\frac{a b^{\prime}}{a^{\prime}}}}, \frac{a^{\prime}}{a} t\right)
\end{aligned}
$$

is a minimizer of the mean distortion in $\mathcal{F}$ for the density $\widetilde{\rho}_{0}$.

2.2. Uniqueness up to rotations of the map. Here, we are dealing with finding every minimizer of the mean distortion in $\mathcal{F}$ for the density $\widetilde{\rho}_{0}$. We will show that such minimizers must be constructed as we did in the previous section. Thus, the section is dedicated to the proof of the following.

Theorem 2.5. Suppose that $f \in \mathcal{F}$ minimizes the mean distortion in $\mathcal{F}$ for the density $\widetilde{\rho}_{0}$. Then, there is $\alpha \in \mathbb{R}$ such that $f=\widetilde{f}_{\alpha}$.

The proof is decomposed in three steps. The first two are a reformulation of the beginning of [BFP15] in the setting of cylinders. In the third one, we prove that, for a minimizer of the mean distortion considered, $f, \Pi \circ f$ does not depend on $\arg (z)$, so $\Pi \circ f$ induces a quasiconformal map $g: R_{a, b} \longrightarrow R_{a^{\prime}, b^{\prime}}$ which will be in fact a minimizer of the mean distortion in $\mathcal{G}$ for the density $\rho_{0}$.

We start by giving a characterisation lemma for curves to be in $\widetilde{\Gamma}_{0}$.

Lemma 2.6. Let $\widetilde{\Gamma}$ be the set of all horizontal curves joining the two boundary discs of $C_{a, b}$ and take an element $\gamma$ of $\widetilde{\Gamma}$. Then,

$$
\int_{\gamma} \widetilde{\rho}_{0} \mathrm{~d} l \geq 1
$$

Moreover, we get equality if and only if $\gamma \in \widetilde{\Gamma}_{0}$. 
Proof. If $\gamma \in \widetilde{\Gamma}$, take a parametrization of $\gamma$ between 0 and $a$,

$$
\begin{aligned}
\int_{\gamma} \rho_{0} \mathrm{~d} l & =\frac{1}{a} \int_{0}^{a} 2\left|\gamma_{1}(s)\right|\left|\dot{\gamma}_{1}(s)\right| \mathrm{d} s \\
& =\frac{1}{a} \int_{0}^{a}\left|\frac{\mathrm{d}}{\mathrm{d} s}\left(\gamma_{2}+i\left|\gamma_{1}\right|^{2}\right)(s)\right| \mathrm{d} s \\
& \geq \frac{1}{a} \int_{0}^{a}\left|\dot{\gamma}_{2}(s)\right| \mathrm{d} s \\
& =1 .
\end{aligned}
$$

Equality happens if and only if $\left|\gamma_{1}\right|^{2}$ is constant. In that case, one may check that $\gamma(s)=\left(z e^{-i \frac{\zeta(s)}{2|z|^{2}}}, \zeta(s)\right)$, meaning $\gamma \in \widetilde{\Gamma}_{0}$

Let $\widetilde{\Gamma}_{0}^{\prime}$ be the curve family

$$
\widetilde{\Gamma}_{0}^{\prime}=\left\{\delta_{z}(s)=\left(z e^{-i \frac{s}{2|z|^{2}}}, s\right)|0<| z \mid<\sqrt{b^{\prime}}\right\} .
$$

Notice that $f \in \mathcal{F}$ minimizes the mean distortion in $\mathcal{F}$ for the density $\widetilde{\rho}_{0}$ if and only if $M\left(\widetilde{\Gamma}_{0}^{\prime}\right)=\int_{C_{a, b}} K(., f)^{2} \widetilde{\rho}_{0}^{4} \mathrm{~d} L^{3}$. Indeed, since $\widetilde{f}_{\alpha}$ minimizes the mean distortion in $\mathcal{F}$ for the density $\widetilde{\rho}_{0}$, any other minimizer $f$ satisfies

$$
\int_{C_{a, b}} K(., f)^{2} \widetilde{\rho}_{0}^{4} \mathrm{~d} L^{3}=\int_{C_{a, b}} K\left(., \widetilde{f}_{\alpha}\right)^{2} \widetilde{\rho}_{0}^{4} \mathrm{~d} L^{3} .
$$

Moreover, we have

$$
M\left(\widetilde{f}_{\alpha}\left(\widetilde{\Gamma}_{0}\right)\right)=M\left(\widetilde{\Gamma}_{0}^{\prime}\right)=\int_{C_{a, b}} K\left(., \widetilde{f}_{\alpha}\right)^{2} \widetilde{\rho}_{0}^{4} \mathrm{~d} L^{3} .
$$

So, $f \in \mathcal{F}$ satisfies $\int_{C_{a, b}} K(., f)^{2} \widetilde{\rho}_{0}^{4} \mathrm{~d} L^{3}=\int_{C_{a, b}} K\left(., \widetilde{f}_{\alpha}\right)^{2} \widetilde{\rho}_{0}^{4} \mathrm{~d} L^{3}$ if and only if $M\left(\widetilde{\Gamma}_{0}^{\prime}\right)$ $=\int_{C_{a, b}} K(., f)^{2} \widetilde{\rho}_{0}^{4} \mathrm{~d} L^{3}$. Then, we prove the following.

Proposition 2.7. If $f$ is as in Theorem 2.5, then $f\left(\widetilde{\Gamma}_{0}\right)=\widetilde{\Gamma}_{0}^{\prime}$, meaning that for every $0<|z|<\sqrt{b}$ and $s \in] 0, a[$,

$$
f\left(\gamma_{z}(s)\right)=\left(z^{\prime} e^{-i \frac{\zeta_{z}(s)}{2\left|z^{\prime}\right|^{2}}}, \zeta_{z}(s)\right)
$$

where $\zeta_{z}$ is a homeomorphism from $] 0, a[$ to $] 0, a^{\prime}[$. Moreover, $f$ maps the vertical axis on the vertical axis.

Proof. The fact that $f$ minimizes the mean distortion in $\mathcal{F}$ for the extremal density $\widetilde{\rho}_{0}$ ensures that $f_{*} \widetilde{\rho}_{0}$ is an extremal density of the family $\widetilde{\Gamma}_{0}^{\prime}$. But,

$$
\widetilde{\rho}_{0}^{\prime}(z, t)=\frac{2|z|}{a^{\prime}}
$$

is also an extremal density of the family $\widetilde{\Gamma}_{0}^{\prime}$. Then, by Lemma 1.3 , since $f_{*} \widetilde{\rho}_{0}$ and $\widetilde{\rho}_{0}^{\prime}$ are continuous,

$$
f_{*} \widetilde{\rho}_{0}=\widetilde{\rho}_{0}^{\prime} .
$$

Let $\delta \in \widetilde{\Gamma}_{0}^{\prime}$. According to the previous lemma, we get

$$
1=\int_{\delta} \widetilde{\rho}_{0}^{\prime} \mathrm{d} l=\int_{\delta} f_{*} \widetilde{\rho}_{0} \mathrm{~d} l=\int_{0}^{a^{\prime}} f_{*} \widetilde{\rho}_{0}(\delta(s))\left|\dot{\delta}_{1}(s)\right| \mathrm{d} s .
$$


Moreover, since $f^{-1}(\delta) \in \Gamma$, we have, using the fact that $\left|Z\left(f^{-1}\right)_{1}\right|+\left|\bar{Z}\left(f^{-1}\right)_{1}\right|=$ $\frac{1}{\left|Z f_{1}\right|-\left|\bar{Z} f_{1}\right|} \circ f^{-1}$ and inequality (3),

$$
\begin{aligned}
1 & \leq \int_{f^{-1}(\delta)} \widetilde{\rho}_{0} \mathrm{~d} l \\
& =\int_{0}^{a^{\prime}} \widetilde{\rho}_{0}\left(f^{-1}(\delta(s))\right)\left|\left(\left(f^{-1}\right)_{1} \circ \delta(s)\right)\right| \mathrm{d} s \\
& \leq \int_{0}^{a^{\prime}} \widetilde{\rho}_{0}\left(f^{-1}(\delta(s))\right)\left|\dot{\delta}_{1}(s)\right|\left(\left|Z\left(f^{-1}\right)_{1}(\delta(s))\right|+\left|\bar{Z}\left(f^{-1}\right)_{1}(\delta(s))\right|\right) \mathrm{d} s \\
& =\int_{0}^{a^{\prime}} f_{*} \widetilde{\rho}_{0}(\delta(s))\left|\dot{\delta}_{1}(s)\right| \mathrm{d} s \\
& =1 .
\end{aligned}
$$

Using the previous lemma, it means that $f^{-1}(\delta) \in \widetilde{\Gamma}_{0}$. So,

$$
C_{a^{\prime}, b^{\prime}} \backslash\{z=0\} \subset f\left(C_{a, b} \backslash\{z=0\}\right) .
$$

Since $f$ is a homeomorphism,

$$
f\left(C_{a, b} \cap\{z=0\}\right) \subset C_{a^{\prime}, b^{\prime}} \cap\{z=0\} .
$$

Moreover, using that $f \in \mathcal{F}$, it implies $f(0,0)=(0,0)$ and $f(0, a)=\left(0, a^{\prime}\right)$. So, $f$ maps the vertical axis to itself and $f\left(\widetilde{\Gamma}_{0}\right)=\widetilde{\Gamma}_{0}^{\prime}$ because $\widetilde{\Gamma}_{0}$ and $\widetilde{\Gamma}_{0}^{\prime}$ are foliations of $C_{a, b} \backslash\{z=0\}$ and $C_{a^{\prime}, b^{\prime}} \backslash\{z=0\}$, respectively.

Now that we know that $f\left(z e^{-i \frac{s}{2|z|^{2}}}, s\right)=\left(z^{\prime} e^{-i \frac{\zeta_{z}(s)}{2\left|z^{\prime}\right|^{2}}}, \zeta_{z}(s)\right)$, we want to find the functions $\zeta_{z}$.

Proposition 2.8. For every $0<|z|<\sqrt{b}$ and $s \in] 0, a[$, we have

$$
f\left(z e^{-i \frac{s}{2|z|^{2}}}, s\right)=\left(z^{\prime} e^{-i \frac{a^{\prime} s}{2 a\left|z^{\prime}\right|^{2}}}, \frac{a^{\prime}}{a} s\right)
$$

for a complex number $0<\left|z^{\prime}\right|<\sqrt{b^{\prime}}$.

Before giving a proof, we need the following result: Proposition 2.12 in BFP15, p. 133]. If $f$ is a map as in Theorem 2.5, then for every curve $\gamma \in \widetilde{\Gamma}_{0}$,

$$
\left|\left(f_{1} \circ \gamma\right)\right|=\left(\left|Z f_{1}(\gamma)\right|-\left|\bar{Z} f_{1}(\gamma)\right|\right)\left|\dot{\gamma}_{1}\right|
$$

This property is called the minimal stretching property.

Proof. In order to prove this, we consider two vector fields on $\mathbf{H} \backslash\{z=0\}$

$$
W:=-\frac{i z}{2|z|^{2}} Z \quad \text { and } \quad \bar{W}:=\frac{i \bar{z}}{2|z|^{2}} \bar{Z} .
$$

Then, $W_{\gamma(s)}=\dot{\gamma}_{1}(s) Z_{\gamma(s)}$ and $\bar{W}_{\gamma(s)}=\dot{\bar{\gamma}}_{1}(s) \bar{Z}_{\gamma(s)}$ for every $\gamma \in \Gamma_{0}$. Thus,

$$
\left|W f_{1}(\gamma)+\bar{W} f_{1}(\gamma)\right|=\left|\dot{\gamma}_{1} Z f_{1}(\gamma)+\dot{\bar{\gamma}}_{1} \bar{Z} f_{1}(\gamma)\right|=\left|\left(f_{1} \circ \gamma\right)\right|
$$

So, using (5), we have

$$
\left|W f_{1}(\gamma)+\bar{W} f_{1}(\gamma)\right|=\left(\left|Z f_{1}(\gamma)\right|-\left|\bar{Z} f_{1}(\gamma)\right|\right)\left|\dot{\gamma}_{1}\right|
$$


Since $\left|Z f_{1}(\gamma)\right|-\left|\bar{Z} f_{1}(\gamma)\right|=\frac{1}{\left|\dot{\gamma}_{1}\right|}\left(\left|W f_{1}(\gamma)\right|-\left|\bar{W} f_{1}(\gamma)\right|\right)$, we get that

$$
\left|W f_{1}+\bar{W} f_{1}\right|=\left|W f_{1}\right|-\left|W f_{1}\right| \cdot
$$

From this and the fact that $f$ is a contact transform, we also deduce

$$
|W(\Pi \circ f)+\bar{W}(\Pi \circ f)|=|W(\Pi \circ f)|-|\bar{W}(\Pi \circ f)|=2\left|f_{1}\right|\left(\left|W f_{1}\right|-\left|\bar{W} f_{1}\right|\right) .
$$

Finally, by definition of $W$ and $\bar{W}$, we find

$$
|W(\Pi \circ f)|-|\bar{W}(\Pi \circ f)|=\frac{\left|f_{1}\right|}{|z|}\left(\left|Z f_{1}\right|-\left|\bar{Z} f_{1}\right|\right) .
$$

Now, we know that

$$
f_{*} \widetilde{\rho}_{0} \circ f=\frac{\widetilde{\rho}_{0}}{\left|Z f_{1}\right|-\left|\bar{Z} f_{1}\right|} \underset{\text { by }(\underline{6})}{=} \frac{2\left|f_{1}\right|}{a} \frac{1}{|W(\Pi \circ f)|-|\bar{W}(\Pi \circ f)|}
$$

and, by continuity and Lemma 1.3 .

$$
f_{*} \widetilde{\rho}_{0} \circ f=\widetilde{\rho}_{0}^{\prime} \circ f=\frac{2\left|f_{1}\right|}{a^{\prime}} .
$$

Thus,

$$
|W(\Pi \circ f)|-|\bar{W}(\Pi \circ f)|=\frac{a^{\prime}}{a} .
$$

Moreover, Proposition 2.7 leads to $\left(\Pi \circ f \circ \gamma_{z}\right)(s)=\zeta_{z}(s)+i\left|z^{\prime}\right|^{2}$ for every curve $\gamma_{z} \in \widetilde{\Gamma}_{0}$. So, $\frac{\mathrm{d}}{\mathrm{d} s} \zeta_{z}(s)=\frac{\mathrm{d}}{\mathrm{d} s}\left(\Pi \circ f \circ \gamma_{z}\right)(s)$. But,

$$
\begin{aligned}
\left|\frac{\mathrm{d}}{\mathrm{d} s}\left(\Pi \circ f \circ \gamma_{z}\right)\right| & =\left|Z(\Pi \circ f)\left(\gamma_{z}\right) \dot{\gamma}_{z, 1}+\bar{Z}(\Pi \circ f)\left(\gamma_{z}\right) \dot{\bar{\gamma}}_{z, 1}\right| \\
& =\left|W(\Pi \circ f)\left(\gamma_{z}\right)+\bar{W}(\Pi \circ f)\left(\gamma_{z}\right)\right| \\
& =\left|W(\Pi \circ f)\left(\gamma_{z}\right)\right|-\left|\bar{W}(\Pi \circ f)\left(\gamma_{z}\right)\right| \\
& =\frac{a^{\prime}}{a} .
\end{aligned}
$$

Thus, $\zeta_{z}(s)=\frac{a^{\prime}}{a} s$ for every $0<|z|<\sqrt{b}$.

In particular, we proved that $f_{2}(z, t)=\frac{a^{\prime}}{a} t$. Now, we are in a position to show that $f_{2}+i\left|f_{1}\right|^{2}$ does not depend on $\arg (z)$.

Proof of Theorem 2.5. As shown previously, it is more convenient to think in cylindrical coordinates. In those coordinates, the curves $\gamma_{z}$ are the curves $s \mapsto(r, \theta-$ $\left.\frac{s}{2 r^{2}}, s\right)$ for $0<r<\sqrt{b}$ and $\theta \in \mathbb{R}$ and we write the map $f$ as $(R, \Theta, T)$ (meaning that $\left.\left(f_{1}, f_{2}\right)=\left(R e^{i \Theta}, T\right)\right)$. Since $f$ maps $\widetilde{\Gamma}_{0}$ to $\widetilde{\Gamma}_{0}^{\prime}$, then

$$
\frac{\mathrm{d}}{\mathrm{d} s} R\left(r, \theta-\frac{s}{2 r^{2}}, s\right)=0 .
$$

Thus,

As it is true for every $r, \theta$, we have

$$
R_{\theta}\left(r, \theta-\frac{s}{2 r^{2}}, s\right)=2 r^{2} R_{t}\left(r, \theta-\frac{s}{2 r^{2}}, s\right) .
$$

$$
\left.R_{\theta}(r, \theta, t)=2 r^{2} R_{t}(r, \theta, t) \text { for every }(r, \theta, t) \in\right] 0, \sqrt{b}[\times \mathbb{R} \times] 0, a[.
$$

Differentiating with respect to $r$, we also find,

$$
R_{r, \theta}(r, \theta, t)=4 r R_{t}(r, \theta, t)+2 r^{2} R_{r, t}(r, \theta, t)
$$


for every $(r, \theta, t) \in] 0, \sqrt{b}[\times \mathbb{R} \times] 0, a\left[\right.$. Since $(R, \Theta, T)$ is a contact map with $T_{r}(r, \theta, t)$ $=0$, then

$$
\Theta_{r}(r, \theta, t)=0 \text {. }
$$

Moreover, there is a nowhere vanishing function $\lambda$ such that for every $(r, \theta, t) \in$ ] $0, \sqrt{b}[\times \mathbb{R} \times] 0, a[$, we have

$$
\frac{a^{\prime}}{a}+2 R^{2}(r, \theta, t) \Theta_{t}(r, \theta, t)=\lambda(r, \theta, t) \text { and } 2 R^{2}(r, \theta, t) \Theta_{\theta}(r, \theta, t)=2 r^{2} \lambda(r, \theta, t) .
$$

This leads to, for every $(r, \theta, t) \in] 0, \sqrt{b}[\times \mathbb{R} \times] 0, a[$,

$$
\Theta_{\theta}(r, \theta, t)=2 r^{2} \Theta_{t}(r, \theta, t)+\frac{a^{\prime} r^{2}}{a R^{2}(r, \theta, t)} .
$$

Now, since $\Theta_{r}=0$, differentiating the previous equation with respect to $r$, we get for every $(r, \theta, t) \in] 0, \sqrt{b}[\times \mathbb{R} \times] 0, a[$,

$$
0=4 r \Theta_{t}(r, \theta, t)+\frac{a^{\prime}}{a}\left(\frac{2 r}{R^{2}(r, \theta, t)}-\frac{2 r^{2} R_{r}(r, \theta, t)}{R^{3}(r, \theta, t)}\right) .
$$

Then, for every $(r, \theta, t) \in] 0, \sqrt{b}[\times \mathbb{R} \times] 0, a[$,

$$
2 \Theta_{t}(r, \theta, t)=\frac{a^{\prime}}{a}\left(\frac{r R_{r}(r, \theta, t)}{R^{3}(r, \theta, t)}-\frac{1}{R^{2}(r, \theta, t)}\right) .
$$

Replacing in (7): for every $(r, \theta, t) \in] 0, \sqrt{b}[\times \mathbb{R} \times] 0, a[$,

$$
\Theta_{\theta}(r, \theta, t)=\frac{a^{\prime} r^{3} R_{r}(r, \theta, t)}{a R^{3}(r, \theta, t)} .
$$

Differentiating the expression of $\Theta_{t}$ with respect to $\theta$, replacing $R_{\theta}(r, \theta, t)$ by $2 r^{2} R_{t}(r, \theta, t)$ and $R_{r, \theta}(r, \theta, t)$ by $4 r R_{t}(r, \theta, t)+2 r^{2} R_{r, t}(r, \theta, t)$, we find that for every $(r, \theta, t) \in] 0, \sqrt{b}[\times \mathbb{R} \times] 0, a[$,

$$
\Theta_{\theta, t}(r, \theta, t)=\frac{a^{\prime} r^{2}}{a}\left(\frac{4 R_{t}(r, \theta, t)+r R_{t, r}(r, \theta, t)}{R^{3}(r, \theta, t)}-\frac{3 r R_{r}(r, \theta, t) R_{t}(r, \theta, t)}{R^{4}(r, \theta, t)}\right) .
$$

Differentiating the expression of $\Theta_{\theta}$ with respect to $t$, we have for every $(r, \theta, t) \in$ ] $0, \sqrt{b}[\times \mathbb{R} \times] 0, a[$

$$
\Theta_{t, \theta}(r, \theta, t)=\frac{a^{\prime} r^{2}}{a}\left(\frac{r R_{t, r}(r, \theta, t)}{R^{3}(r, \theta, t)}-\frac{3 r R_{r}(r, \theta, t) R_{t}(r, \theta, t)}{R^{4}(r, \theta, t)}\right) .
$$

Since we assumed all our maps to be $C^{2}$, by use of the Schwarz theorem about commutativity of partial derivatives, we conclude that for every $(r, \theta, t) \in] 0, \sqrt{b}[\times \mathbb{R} \times] 0, a[$,

$$
\frac{4 R_{t}(r \theta, t)}{R^{3}(r, \theta, t)}=0
$$

This leads to

$$
R_{t}(r, \theta, t)=R_{\theta}(r, \theta, t)=0
$$

for every $(r, \theta, t) \in] 0, \sqrt{b}[\times \mathbb{R} \times] 0, a[$. Then, $f$ must be constructed as a lift of a quasiconformal map $g$ between rectangles. Now, it remains to show that $g$ is a minimizer of the mean distortion in $\mathcal{G}$ for the density $\rho_{0}$.

First, notice that

$$
M\left(f\left(\widetilde{\Gamma}_{0}\right)\right)=\int_{C_{a, b}} K(., f)^{2} \widetilde{\rho}_{0}^{4} \mathrm{~d} L^{3} \text { and } \widetilde{\rho}_{0}=\Pi^{*} \rho_{0} .
$$


Moreover, if $\Gamma_{0}^{\prime}=\Pi\left(f\left(\widetilde{\Gamma}_{0}\right)\right)$, then by Proposition 2.7. $\Gamma_{0}^{\prime}$ is the family of straight horizontal lines in the rectangle $R_{a^{\prime}, b^{\prime}}$ which has as extremal density $\rho_{0}^{\prime}=\frac{1}{a^{\prime}}$ and its lifted family is by definition $\widetilde{\Gamma}_{0}^{\prime}$ which has as extremal density $\Pi^{*} \rho_{0}^{\prime}$. We can then apply Corollary 1.13 to state that

$$
M\left(g\left(\Gamma_{0}\right)\right)=\int_{R_{a, b}} K(., g) \rho_{0}^{2} \mathrm{~d} L^{2} .
$$

Finally, let $\Gamma$ be the set of all curves in $R_{a, b}$ connecting the two vertical boundary edges. Then, $\rho_{0} \in \operatorname{adm}(\Gamma)$ and for every $h \in \mathcal{G}$, since $g\left(\Gamma_{0}\right) \subset h(\Gamma), M\left(g\left(\Gamma_{0}\right)\right) \leq$ $M(h(\Gamma))$. So, by a result similar to Proposition 1.6 for quasiconformal maps in the complex plane, $g$ minimizes the mean distortion in $\mathcal{G}$ for the density $\rho_{0}$. Thus, by Lemma 2.2 and Proposition 2.3. $f$ is one of the $\widetilde{f}_{\alpha}$.

To sum up, in Section 2 we proved the following.

Theorem 2.9. The map

$$
\begin{aligned}
& \tilde{f}_{0}: \quad C_{a, b} \quad \longrightarrow \quad C_{a^{\prime}, b^{\prime}} \\
& (z, t) \longmapsto\left(\frac{\sqrt{b^{\prime}} z e^{\frac{i}{2 b}\left(1-\frac{a^{\prime} b}{a b^{\prime}}\right) t}}{\sqrt{\left(1-\frac{a b^{\prime}}{a^{\prime} b}\right)|z|^{2}+\frac{a b^{\prime}}{a^{\prime}}}}, \frac{a^{\prime}}{a} t\right)
\end{aligned}
$$

minimizes the mean distortion in $\mathcal{F}$ for the density $\widetilde{\rho}_{0}$ and is the unique such minimizer up to composition with a rotation around the vertical axis.

\section{Generalized Construction}

3.1. Notation and the "pull-back density condition". In this section, we want to determine conditions in order to generalize the construction we made before to domains in $\mathbf{H}$ that are not conformally equivalent to cylinders but whose projections on the half-plane are biholomorphic to rectangles. So, let us take two bounded domains of $\mathbb{H}, \Omega_{a, b}$ and $\Omega_{a^{\prime}, b^{\prime}}$ with two biholomorphic maps $\phi: R_{a, b} \longrightarrow \Omega_{a, b}$ and $\psi: R_{a^{\prime}, b^{\prime}} \longrightarrow \Omega_{a^{\prime}, b^{\prime}}$ that extend homeomorphically to boundaries (boundaries taken in $\mathbb{C}$ ). $\Omega_{a, b}$ and $\Omega_{a^{\prime}, b^{\prime}}$ may also be defined as quadrilaterals in the sense of Ahlfors (see Ahl66]).

Notation 3.1.

- We denote $\Gamma_{0}$ (resp., $\Gamma_{0}^{\prime}$ ) the family of horizontal lines in $R_{a, b}\left(\operatorname{resp} ., R_{a^{\prime}, b^{\prime}}\right)$, then $\Gamma_{\phi}:=\phi\left(\Gamma_{0}\right)$ and $\Gamma_{\psi}:=\psi\left(\Gamma_{0}^{\prime}\right)$.

- We also denote $\rho_{0}=\frac{1}{a}$ (resp., $\rho_{0}^{\prime}=\frac{1}{a^{\prime}}$ ) the extremal density of $\Gamma_{0}$ (resp., $\left.\Gamma_{0}^{\prime}\right)$. Then, $\rho_{\phi}=\phi_{*} \rho_{0} \in \operatorname{adm}\left(\Gamma_{\phi}\right)$ and $\rho_{\psi}=\psi_{*} \rho_{0}^{\prime} \in \operatorname{adm}\left(\Gamma_{\psi}\right)$ are the push-forward densities. Since $\phi$ and $\psi$ are holomorphic, $\rho_{\phi}$ (resp., $\rho_{\psi}$ ) is extremal for $\Gamma_{\phi}$ (resp., $\Gamma_{\psi}$ ).

- Consider $R_{a, b}$ and $R_{a^{\prime}, b^{\prime}}$ as $] 0, a[\times] 0, b[$ and $] 0, a^{\prime}[\times] 0, b^{\prime}$ [ and still write $\phi:] 0, a[\times] 0, b\left[\longrightarrow \Omega_{a, b}\right.$ and $\left.\psi:\right] 0, a^{\prime}[\times] 0, b^{\prime}\left[\longrightarrow \Omega_{a^{\prime}, b^{\prime}}\right.$ for the holomorphic maps. Then, for every $s \in[0, a], \partial \Omega_{s}:=\phi(\{s\} \times[0, b])$ and for every $s \in\left[0, a^{\prime}\right], \partial \Omega_{s}^{\prime}:=\psi\left(\{s\} \times\left[0, b^{\prime}\right]\right)$.

- We denote $\mathcal{G}_{\phi, \psi}$ the set of quasiconformal maps from $\Omega_{a, b}$ to $\Omega_{a^{\prime}, b^{\prime}}$ that extend homeomorphically to boundaries and map $\partial \Omega_{0}$ to $\partial \Omega_{0}^{\prime}$ and $\partial \Omega_{a}$ to $\partial \Omega_{a^{\prime}}^{\prime}$ 
Remark 3.2. Notice that since quasiconformal maps (in $\mathbb{C}$ ) are orientation-preserving, $\mathcal{G}$ can also be described as the set of quasiconformal maps from $R_{a, b}$ to $R_{a^{\prime}, b^{\prime}}$ that extend homeomorphically to closures and map the vertical boundary components onto their corresponding ones. In other words, $\mathcal{G}_{i d, i d}=\mathcal{G}$.

Recall from Lemma 2.2 that every minimizer of the mean distortion in $\mathcal{G}$ for the density $\rho_{0}$ is of the form

$$
f_{\varphi}(x+i y)=\frac{a^{\prime}}{a} x+i \varphi(y)
$$

for a function $\varphi:[0, b] \longrightarrow\left[0, b^{\prime}\right]$ with $\varphi(0)=0, \varphi(b)=b^{\prime}$ and $\dot{\varphi} \geq \frac{a^{\prime}}{a}$. Now, since the modulus of a curve family is a conformal invariant, every minimizer of the mean distortion in $\mathcal{G}_{\phi, \psi}$ for the density $\rho_{\phi}$ is one of the

$$
g_{\varphi}=\psi \circ f_{\varphi} \circ \phi^{-1} \text {. }
$$

In order to state the results of the section, we need to introduce more notation.

Notation 3.3.

- $\widetilde{\Omega}_{a, b}:=\overline{\Psi\left(S^{1} \times \Omega_{a, b}\right)}$ and $\widetilde{\Omega}_{a^{\prime}, b^{\prime}}:=\overline{\Psi\left(S^{1} \times \Omega_{a^{\prime}, b^{\prime}}\right)}$. These are the right domains to consider since, as in cylinders, we don't want potential pieces of the vertical axis to be on the topological boundaries.

- $\widetilde{\Gamma}_{\phi}$ (resp., $\left.\widetilde{\Gamma}_{\psi}\right)$ is the lifted family of $\Gamma_{\phi}\left(\right.$ resp., $\left.\Gamma_{\psi}\right)$.

- Extending $\Pi$ to a function from $\mathbf{H}$ to $\mathbb{H} \cup\{\Im(w)=0\}$, we write for every $s \in[0, a], \partial \widetilde{\Omega}_{s}=\Pi^{-1}\left(\partial \Omega_{s}\right)$ and for every $s \in\left[0, a^{\prime}\right], \partial \widetilde{\Omega}_{s}^{\prime}=\Pi^{-1}\left(\partial \Omega_{s}^{\prime}\right)$.

- The set of quasiconformal maps from $\widetilde{\Omega}_{a, b}$ to $\widetilde{\Omega}_{a^{\prime}, b^{\prime}}$ that extend homeomorphically to boundaries and map $\partial \widetilde{\Omega}_{0}$ to $\partial \widetilde{\Omega}_{0}^{\prime}$ and $\partial \widetilde{\Omega}_{a}$ to $\partial \widetilde{\Omega}_{a^{\prime}}^{\prime}$ is denoted $\mathcal{F}_{\phi, \psi}$. Notice that $\mathcal{F}_{i d, i d}$ is the set $\mathcal{F}$ from Section 2

Notice that it could happen that $\partial \widetilde{\Omega}_{s}$ lies inside $\widetilde{\Omega}_{a, b}$ for $s \in\{0, a\}$ and not in the topological boundary of $\widetilde{\Omega}_{a, b}$.

In the whole section, we will assume that $\Pi^{*} \rho_{\phi}$ (resp., $\Pi^{*} \rho_{\psi}$ ) is extremal for $\widetilde{\Gamma}_{\phi}$ (resp., $\widetilde{\Gamma}_{\phi}$ ). This is a quite important condition for both existence and uniqueness results of Section 3. We wish to give a better understanding of this condition. First, let us give an example of a holomorphic map $\phi$ for which $\Pi^{*} \rho_{\phi}$ isn't extremal for the family $\widetilde{\Gamma}_{\phi}$.

Example 3.4. The example is quite simple. We know that in a rectangle $R_{a, b}$, horizontal lines satisfy the above property, but vertical ones don't. Indeed, let $\Delta$ be the family of curves

$$
\delta_{x}(s)=x+i s \text { for } 0<x<a \text { and } 0<s<b .
$$

It is well known that the modulus of the family $\Delta$ is $\frac{a}{b}$ with extremal density $\sigma=\frac{1}{b}$. Now, let $\widetilde{\Delta}$ be the lifted family of $\Delta$. One may verify that $\widetilde{\Delta}$ is the family of curves

$$
\widetilde{\delta}_{(z, t)}(s)=(s z, t) \text { for }|z|=1,0<t<a \text { and } 0<s<\sqrt{b} .
$$

With a calculus quite similar to the one done in the proof of Lemma 2.1, we find the modulus of $\widetilde{\Delta}$ to be

$$
M(\widetilde{\Delta})=\frac{16 \pi a}{27 b}
$$


with extremal density

$$
\widetilde{\sigma}(z, t)=\frac{2}{3 b^{\frac{1}{3}}|z|^{\frac{1}{3}}}
$$

which is not the pull-back by $\Pi$ of $\sigma$. Now, we may send the rectangle $R_{b, a}$ to the rectangle $R_{a, b}$ by the composition of a rotation of angle $\frac{\pi}{2}$ and a translation by $a$ which is a holomorphic map sending horizontal lines to vertical ones.

The following gives an interpretation of the pull-back density condition in our setting ( $h^{\prime}$ refers to the complex derivative of any holomorphic function $h$ ).

Proposition 3.5. If $\frac{\left|\phi^{\prime}\right|}{\Im(\phi)}$ is a function of the imaginary part only, then

$$
M\left(\widetilde{\Gamma}_{\phi}\right)=\int_{\widetilde{\Omega}_{a, b}}\left(\Pi^{*} \rho_{\phi}\right)^{4} \mathrm{~d} L^{3} .
$$

Conversely, if $M\left(\widetilde{\Gamma}_{\phi}\right)=\int_{\widetilde{\Omega}_{a, b}}\left(\Pi^{*} \rho_{\phi}\right)^{4} \mathrm{~d} L^{3}$, then $\frac{\left|\phi^{\prime}\right|}{\Im(\phi)}$ is a function of the imaginary part only.

Proof. Let $\gamma_{x, \alpha} \in \widetilde{\Gamma}_{\phi}$. By definition,

$$
\gamma_{x, \alpha}(s)=\left(\sqrt{\Im(\phi(s, x))} e^{i(\alpha+\tau(s, x))}, \Re(\phi(s, x))\right)
$$

with $\tau(s, x)=-\int_{0}^{s} \frac{\Re\left(\phi_{t}(t, x)\right)}{2 \Im(\phi(t, x))} \mathrm{d} t$ for every $s, x$ (here, the index $t$ refers to the partial derivative with respect to the first variable). Then, computing, we have for every $s, x$

$$
\left|\dot{\gamma}_{x, \alpha, 1}(s)\right|=\frac{\left|\phi^{\prime}(s, x)\right|}{2 \sqrt{\Im(\phi(s, x))}},
$$

where $\gamma_{x, \alpha, 1}$ is the first coordinate of the curve $\gamma_{x, \alpha}$. Thus, if $\rho \in \operatorname{adm}\left(\widetilde{\Gamma}_{\phi}\right)$, then, for every $x$, we have

$$
\int_{0}^{a} \rho\left(\gamma_{x, \alpha}(s)\right) \frac{\left|\phi^{\prime}(s, x)\right|}{2 \sqrt{\Im(\phi(s, x))}} \mathrm{d} s \geq 1 .
$$

But, by substitution, we have the following:

$$
\int_{\widetilde{\Omega}_{a, b}} \rho^{4} \mathrm{~d} L^{3}=\frac{1}{2} \int_{0}^{2 \pi} \int_{0}^{b} \int_{0}^{a} \rho^{4}\left(\gamma_{x, \alpha}(s)\right)\left|\phi^{\prime}(s, x)\right|^{2} \mathrm{~d} s \mathrm{~d} x \mathrm{~d} \alpha .
$$

Moreover, using Hölder inequality, we have for every $x, \alpha$,

$$
\begin{aligned}
\left(\int_{0}^{a} \rho\left(\gamma_{x, \alpha}(s)\right) \frac{\left|\phi^{\prime}(s, x)\right|}{2 \sqrt{\Im(\phi(s, x))}} \mathrm{d} s\right)^{4} \leq\left(\int_{0}^{a} \rho^{4}\left(\gamma_{x, \alpha}(s)\right)\left|\phi^{\prime}(s, x)\right|^{2} \mathrm{~d} s\right) & \\
& \times\left(\int_{0}^{a}\left(\frac{\left|\phi^{\prime}(s, x)\right|^{\frac{1}{2}}}{2 \sqrt{\Im(\phi(s, x))}}\right)^{\frac{4}{3}} \mathrm{~d} s\right)^{3} .
\end{aligned}
$$

So that

$$
1 \leq \int_{0}^{a} \rho^{4}\left(\gamma_{x, \alpha}(s)\right)\left|\phi^{\prime}(s, x)\right|^{2} \mathrm{~d} s\left(\int_{0}^{a}\left(\frac{\left|\phi^{\prime}(s, x)\right|^{\frac{1}{2}}}{2 \sqrt{\Im(\phi(s, x))}}\right)^{\frac{4}{3}} \mathrm{~d} s\right)^{3} .
$$


Now, assume first that $\frac{\left|\phi^{\prime}\right|}{\Im(\phi)}$ is a function of $x$ only. Then by assumption there is a real valued function $h$ such that

$$
h(x)=\frac{(\Im(\phi(s, x)))^{2}}{\left|\phi^{\prime}(s, x)\right|^{2}} \text { for every } s, x .
$$

Thus, using (9), we have for every $x$,

$$
\int_{0}^{a} \rho^{4}\left(\gamma_{x, \alpha}(s)\right)\left|\phi^{\prime}(s, x)\right|^{2} \mathrm{~d} s \geq \frac{16 h(x)}{a^{3}} .
$$

Using (8), it leads to,

Now, by definition,

$$
M\left(\widetilde{\Gamma}_{\phi}\right) \geq \frac{16 \pi}{a^{3}} \int_{0}^{b} h(x) \mathrm{d} x
$$

So, again by substitution,

$$
\Pi^{*} \rho_{\phi}=\frac{|Z \Pi|}{a\left|\phi^{\prime}\left(\phi^{-1} \circ \Pi\right)\right|}
$$

$$
\int_{\widetilde{\Omega}_{a, b}}\left(\Pi^{*} \rho_{\phi}\right)^{4} \mathrm{~d} L^{3}=\frac{1}{2} \int_{0}^{2 \pi} \int_{0}^{b} \int_{0}^{a} \frac{16(\Im(\phi(s, x)))^{2}}{a^{4}\left|\phi^{\prime}(s, x)\right|^{2}} \mathrm{~d} s \mathrm{~d} x \mathrm{~d} \alpha=\frac{16 \pi}{a^{3}} \int_{0}^{b} h(x) \mathrm{d} x .
$$

Thus,

$$
M\left(\widetilde{\Gamma}_{\phi}\right)=\int_{\widetilde{\Omega}_{a, b}}\left(\Pi^{*} \rho_{\phi}\right)^{4} \mathrm{~d} L^{3} .
$$

Conversely, assume that $M\left(\widetilde{\Gamma}_{\phi}\right)=\int_{\widetilde{\Omega}_{a, b}}\left(\Pi^{*} \rho_{\phi}\right)^{4} \mathrm{~d} L^{3}$. Then, using (8) and (9), we find for every $\rho \in \operatorname{adm}\left(\widetilde{\Gamma}_{\phi}\right)$,

$$
\int_{\widetilde{\Omega}_{a, b}} \rho^{4} \mathrm{~d} L^{3} \geq 16 \pi \int_{0}^{b} \frac{\mathrm{d} x}{\left(\int_{0}^{a} \frac{\left|\phi^{\prime}(s, x)\right|^{\frac{2}{3}}}{(\Im(\phi(s, x)))^{\frac{2}{3}}} \mathrm{~d} s\right)^{3}} .
$$

Now, let $\rho \in \operatorname{adm}\left(\widetilde{\Gamma}_{\phi}\right)$ be defined for every $x, s, \alpha$ by

$$
\rho\left(\gamma_{x, \alpha}(s)\right):=\left(\int_{0}^{a} \frac{\left|\phi^{\prime}(s, x)\right|^{\frac{2}{3}}}{2(\Im(\phi(s, x)))^{\frac{2}{3}}} \mathrm{~d} s\right)^{-1} \frac{\left|\phi^{\prime}(s, x)\right|^{-\frac{1}{3}}}{(\Im(\phi(s, x)))^{\frac{1}{6}}} .
$$

Then,

$$
\int_{\widetilde{\Omega}_{a, b}} \rho^{4} \mathrm{~d} L^{3}=16 \pi \int_{0}^{b} \frac{\mathrm{d} x}{\left(\int_{0}^{a} \frac{\left|\phi^{\prime}(s, x)\right|^{\frac{2}{3}}}{(\Im(\phi(s, x)))^{\frac{2}{3}}} \mathrm{~d} s\right)^{3}} .
$$

Since, $\Pi^{*} \rho_{\phi}$ is extremal, we have $\Pi^{*} \rho_{\phi}=\rho$. This leads to

$$
\frac{\left|\phi^{\prime}(s, x)\right|^{\frac{2}{3}}}{(\Im(\phi(s, x)))^{\frac{2}{3}}}=\frac{1}{a} \int_{0}^{a} \frac{\left|\phi^{\prime}(s, x)\right|^{\frac{2}{3}}}{(\Im(\phi(s, x)))^{\frac{2}{3}}} \mathrm{~d} s
$$

for every $s, x$. So, $\frac{\left|\phi^{\prime}\right|}{\Im(\phi)}$ does not depend on $s$.

To end the discussion on the pull-back density condition, one can show that (by the previous proposition) it is equivalent to the condition given on the foliation in Theorem 1.7

In the following, we will answer (in this setting) the questions asked in the Introduction. First, we will give conditions on the function $\varphi$ so that $g_{\varphi}$ lifts to a 
quasiconformal map between $\widetilde{\Omega}_{a, b} \backslash\{z=0\}$ and $\widetilde{\Omega}_{a^{\prime}, b^{\prime}} \backslash\{z=0\}$. To be more precise, we will prove that $g_{\varphi}$ can be lifted to a quasiconformal map between $\widetilde{\Omega}_{a, b} \backslash\{z=$ $0\}$ and $\widetilde{\Omega}_{a^{\prime}, b^{\prime}} \backslash\{z=0\}$ if and only if $\varphi$ satisfies an ordinary differential equation. Moreover, we will see that when such a $\varphi$ can be found and if lifts of $g_{\varphi}$ can be extended to maps in $\mathcal{F}_{\phi, \psi}$, then those lifts minimize the mean distortion in $\mathcal{F}_{\phi, \psi}$ for the density $\Pi^{*} \rho_{\phi}$. After that, we will explain that if we can find a minimizer of the mean distortion functional by that process, then we actually found all of them. Finally, we give two examples.

3.2. Conditions for existence of a lift. As stated, here we wish to find conditions on $\varphi$ so that $g_{\varphi}$ can be lifted by $\Pi$ into a quasiconformal map between $\widetilde{\Omega}_{a, b} \backslash\{z=0\}$ and $\widetilde{\Omega}_{a^{\prime}, b^{\prime}} \backslash\{z=0\}$. To do so, we make a change of coordinates in $\mathbf{H}$ more adapted to the problem. New coordinates are then given by the following two maps:

$$
\begin{array}{cccc}
\left.\Psi_{\phi}: \quad\right] 0, a[\times] 0, b[\times \mathbb{R} & \longrightarrow & \widetilde{\Omega}_{a, b} \backslash\{z=0\} \\
(s, x, \theta) & \longrightarrow & \left(\sqrt{\Im(\phi(s, x))} e^{i \theta}, \Re(\phi(s, x))\right) \\
\left.\Psi_{\psi}: \quad\right] 0, a^{\prime}[\times] 0, b^{\prime}[\times \mathbb{R} & \longrightarrow & \widetilde{\Omega}_{a^{\prime}, b^{\prime}} \backslash\{z=0\} \\
(s, x, \theta) & \longrightarrow & \left(\sqrt{\Im(\psi(s, x))} e^{i \theta}, \Re(\psi(s, x))\right)
\end{array}
$$

so that

$$
\Pi \circ \Psi_{\phi}(s, x, \theta)=\phi(s, x) \quad \text { and } \quad \Pi \circ \Psi_{\psi}(s, x, \theta)=\psi(s, x),
$$

where it makes sense. Moreover, a map $(S, X, \Theta):] 0, a[\times] 0, b[\times \mathbb{R} \longrightarrow] 0, a^{\prime}[\times] 0, b^{\prime}[\times \mathbb{R}$ induces a well-defined map $\left(g_{1}, g_{2}\right): \widetilde{\Omega}_{a, b} \backslash\{z=0\} \longrightarrow \widetilde{\Omega}_{a^{\prime}, b^{\prime}} \backslash\{z=0\}$ if and only if $S, X$ are $2 \pi$-periodic (in the third variable) and $\Theta$ is $2 \pi$-periodic modulo $2 \pi$ (also in the third variable). Then, one may verify that a map $(S, X, \Theta)$ defines a contact map from $\widetilde{\Omega}_{a, b} \backslash\{z=0\}$ to $\widetilde{\Omega}_{a^{\prime}, b^{\prime}} \backslash\{z=0\}$ if and only if there is a nowhere vanishing function $\lambda$ such that,

$$
\begin{aligned}
\Re\left(\psi_{s}(S, X)\right) \mathrm{d} S-\Im\left(\psi_{s}(S, X)\right) \mathrm{d} X+ & 2 \Im(\psi(S, X)) \mathrm{d} \Theta \\
& =\lambda\left(\Re\left(\phi_{s}\right) \mathrm{d} s-\Im\left(\phi_{s}\right) \mathrm{d} x+2 \Im(\phi) \mathrm{d} \theta\right) .
\end{aligned}
$$

Here again, we denote the partial derivative with respect to $s$ (resp., $x$, resp., $\theta$ ) by an index $s$ (resp., $x$, resp., $\theta$ ). Finally, a map $(S, X, \Theta)$ defines a lift of a $g_{\varphi}$ if the diagram

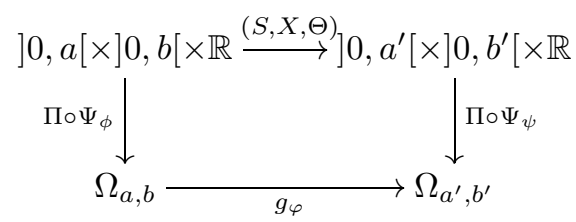

is commutative.

Lemma 3.6. Let $(S, X, \Theta):] 0, a[\times] 0, b[\times \mathbb{R} \longrightarrow] 0, a^{\prime}[\times] 0, b^{\prime}[\times \mathbb{R}$. The previous diagram is commutative if and only if we have

$$
S(s, x, \theta)=S(s)=\frac{a^{\prime}}{a} s \quad \text { and } \quad X(s, x, \theta)=X(x)=\varphi(x)
$$

for every $(s, x, \theta) \in] 0, a[\times] 0, b[\times \mathbb{R}$. In other words, $(s, x) \longmapsto(S(s), X(x))$ agrees with $f_{\varphi}$. 
Proof. The diagram is commutative if and only if

$$
\left(g_{\varphi} \circ \Pi \circ \Psi_{\phi}\right)(s, x, \theta)=\left(\Pi \circ \Psi_{\psi}\right)(S(s, x, \theta), X(s, x, \theta), \Theta(s, x, \theta))
$$

for every $(s, x, \theta) \in] 0, a[\times] 0, b[\times \mathbb{R}$, leading to

$$
(S, X)(s, x, \theta)=\left(\psi^{-1} \circ g_{\varphi} \circ \phi\right)(s, x)=f_{\varphi}(s, x)=\left(\frac{a^{\prime}}{a} s, \varphi(x)\right)
$$

for every $(s, x, \theta) \in] 0, a[\times] 0, b[\times \mathbb{R}$.

To sum up, a map $(S, X, \Theta):] 0, a[\times] 0, b[\times \mathbb{R} \longrightarrow] 0, a^{\prime}[\times] 0, b^{\prime}[\times \mathbb{R}$ induces a contact lift of $g_{\varphi}$ if and only if, $(s, x) \longmapsto(S(s), X(x))$ agrees with $f_{\varphi}, \Theta$ is $2 \pi$-periodic modulo $2 \pi$ in the third variable, and the functions $S, X$, and $\Theta$ satisfy the contact condition (10). Now, we are able to state conditions for the existence of a contact lift; then quasiconformality of the lift comes from Lemma 1.14.

Proposition 3.7. Suppose that $(S, X, \Theta)$ is a contact transform from $] 0, a[\times] 0, b[\times \mathbb{R}$ to $] 0, a^{\prime}[\times] 0, b^{\prime}[\times \mathbb{R}$ such that

$$
g_{\varphi} \circ \Pi \circ \Psi_{\phi}=\Pi \circ \Psi_{\psi} \circ(S, X, \Theta) .
$$

Then, first, for every $(s, x, \theta) \in] 0, a[\times] 0, b[\times \mathbb{R}$,

$$
S(s, x, \theta)=\frac{a^{\prime}}{a} s \text { and } X(s, x, \theta)=\varphi(x) .
$$

Moreover, $\varphi, \psi$, and $\phi$ satisfy for every $(s, x, \theta) \in] 0, a[\times] 0, b[\times \mathbb{R}$

$$
\frac{a^{\prime}}{a} \dot{\varphi}(x) \frac{\left|\psi^{\prime}\left(\frac{a^{\prime}}{a} s, \varphi(x)\right)\right|^{2}(\Im(\phi(s, x)))^{2}}{\left|\phi^{\prime}(s, x)\right|^{2}\left(\Im\left(\psi\left(\frac{a^{\prime}}{a} s, \varphi(x)\right)\right)\right)^{2}}=1 .
$$

Conversely, if those conditions are satisfied, then $(S, X, \Theta):(s, x, \theta) \longmapsto\left(\frac{a^{\prime}}{a} s, \varphi(x)\right.$, $\theta+h(s, x))$ with

$$
\begin{aligned}
2 h_{x}(s, x) & =\frac{\Im\left(\psi_{s}\left(\frac{a^{\prime}}{a} s, \varphi(x)\right)\right)}{\Im\left(\psi\left(\frac{a^{\prime}}{a} s, \varphi(x)\right)\right)} \dot{\varphi}-\frac{\Im\left(\phi_{s}(s, x)\right)}{\Im(\phi(s, x))}, \\
2 h_{s}(s, x) & =\frac{\Re\left(\phi_{s}(s, x)\right)}{\Im(\phi(s, x))}-\frac{a^{\prime}}{a} \frac{\Re\left(\psi_{s}\left(\frac{a^{\prime}}{a} s, \varphi(x)\right)\right)}{\Im\left(\psi\left(\frac{a^{\prime}}{a} s, \varphi(x)\right)\right)},
\end{aligned}
$$

a contact transform satisfying $g_{\varphi} \circ \Pi \circ \Psi_{\phi}=\Pi \circ \Psi_{\psi} \circ(S, X, \Theta)$.

Proof. The proof is similar to the one of 2.3, we take information from the map $(S, X, \Theta)$ to be contact in order to find the three partial derivatives of $\Theta$. According to the previous lemma, we know that $S(s, x, \theta)=\frac{a^{\prime}}{a} s$ and $X(s, x, \theta)=\varphi(x)$ for every $(s, x, \theta) \in] 0, a[\times] 0, b[\times \mathbb{R}$. Now, the contact condition gives the following PDEs:

$$
\begin{aligned}
\frac{a^{\prime}}{a} \Re\left(\psi_{s}(S, X)\right)+2 \Im(\psi(S, X)) \Theta_{s} & =\lambda \Re\left(\phi_{s}\right), \\
\Im\left(\psi_{s}(S, X)\right) \dot{\varphi}-2 \Im(\psi(S, X)) \Theta_{x} & =\lambda \Im\left(\phi_{s}\right), \\
\Im(\psi(S, X)) \Theta_{\theta} & =\lambda \Im(\phi),
\end{aligned}
$$

for a nowhere vanishing function $\lambda$. From (16), since $\Im(\phi)>0$, we find

$$
\lambda=\frac{\Im(\psi(S, X))}{\Im(\phi)} \Theta_{\theta} .
$$


Replacing in (14) and (15) and dividing by $\Im(\psi(S, X))>0$, we deduce

$$
\begin{aligned}
\frac{a^{\prime}}{a} \frac{\Re\left(\psi_{s}(S, X)\right)}{\Im(\psi(S, X))}+2 \Theta_{s} & =\frac{\Re\left(\phi_{s}\right)}{\Im(\phi)} \Theta_{\theta}, \\
\frac{\Im\left(\psi_{s}(S, X)\right)}{\Im(\psi(S, X))} \dot{\varphi}-2 \Theta_{x} & =\frac{\Im\left(\phi_{s}\right)}{\Im(\phi)} \Theta_{\theta} .
\end{aligned}
$$

Differentiating (17) with respect to $x$, (18) with respect to $s$, and using CauchyRiemann equations for $\psi$ and $\phi$, we find

$$
\begin{aligned}
-\frac{a^{\prime}}{a} \dot{\varphi}\left(\frac{\Im\left(\psi_{s, s}(S, X)\right)}{\Im(\psi(S, X))}+\left(\frac{\Re\left(\psi_{s}(S, X)\right)}{\Im(\psi(S, X))}\right)^{2}\right) & +2 \Theta_{x, s}=\frac{\Re\left(\phi_{s}\right)}{\Im(\phi)} \Theta_{x, \theta} \\
& -\left(\frac{\Im\left(\phi_{s, s}\right)}{\Im(\phi)}+\left(\frac{\Re\left(\phi_{s}\right)}{\Im(\phi)}\right)^{2}\right) \Theta_{\theta}, \\
\frac{a^{\prime}}{a} \dot{\varphi}\left(\frac{\Im\left(\psi_{s, s}(S, X)\right)}{\Im(\psi(S, X))}-\left(\frac{\Im\left(\psi_{s}(S, X)\right)}{\Im(\psi(S, X))}\right)^{2}\right) & -2 \Theta_{s, x}=\frac{\Im\left(\phi_{s}\right)}{\Im(\phi)} \Theta_{s, \theta} \\
& +\left(\frac{\Im\left(\phi_{s, s}\right)}{\Im(\phi)}-\left(\frac{\Im\left(\phi_{s}\right)}{\Im(\phi)}\right)^{2}\right) \Theta_{\theta} .
\end{aligned}
$$

Thus, replacing the value of $2 \Theta_{s, x}$ from (19) in (20), and using $\left|h^{\prime}\right|^{2}=\left(\Re\left(h_{s}\right)\right)^{2}+$ $\left(\Im\left(h_{s}\right)\right)^{2}$ for any holomorphic function, we find

$$
-\frac{a^{\prime}}{a} \dot{\varphi} \frac{\left|\psi^{\prime}(S, X)\right|^{2}}{(\Im(\psi(S, X)))^{2}}-\frac{\Re\left(\phi_{s}\right)}{\Im(\phi)} \Theta_{x, \theta}+\frac{\left|\phi^{\prime}\right|^{2}}{(\Im(\phi))^{2}} \Theta_{\theta}=\frac{\Im\left(\phi_{s}\right)}{\Im(\phi)} \Theta_{s, \theta} .
$$

Now, differentiating (17) and (18) both with respect to $\theta$, we also have

$$
\Theta_{s, \theta}=\frac{\Re\left(\phi_{s}\right)}{2 \Im(\phi)} \Theta_{\theta, \theta} \quad \text { and } \quad \Theta_{x, \theta}=-\frac{\Im\left(\phi_{s}\right)}{2 \Im(\phi)} \Theta_{\theta, \theta} .
$$

So, replacing those in (21), we finally have

$$
\Theta_{\theta}=\frac{a^{\prime}}{a} \dot{\varphi}(x) \frac{\left|\psi^{\prime}\left(\frac{a^{\prime}}{a} s, \varphi(x)\right)\right|^{2}(\Im(\phi(s, x)))^{2}}{\left|\phi^{\prime}(s, x)\right|^{2}\left(\Im\left(\psi\left(\frac{a^{\prime}}{a} s, \varphi(x)\right)\right)\right)^{2}} .
$$

The term on the right side does not depend on $\theta$. So

$$
0=\Theta_{\theta, \theta}=\Theta_{s, \theta}=\Theta_{x, \theta} .
$$

Thus, $\Theta_{\theta}$ is constant. So, for $\Theta$ to follow the periodicity condition, $\Theta_{\theta}$ must be everywhere equal to 1 , which ends the first part of the proof.

For the second part, it is a simple verification that a map $(S, X, \Theta)$ defined by $(s, x, \theta) \longmapsto\left(\frac{a^{\prime}}{a} s, \varphi(x), \theta+h(s, x)\right)$ with

$$
\begin{aligned}
2 h_{x}(s, x) & =\frac{\Im\left(\psi_{s}\left(\frac{a^{\prime}}{a} s, \varphi(x)\right)\right)}{\Im\left(\psi\left(\frac{a^{\prime}}{a} s, \varphi(x)\right)\right)} \dot{\varphi}(x)-\frac{\Im\left(\phi_{s}(s, x)\right)}{\Im(\phi(s, x))}, \\
2 h_{s}(s, x) & =\frac{\Re\left(\phi_{s}(s, x)\right)}{\Im(\phi(s, x))}-\frac{a^{\prime}}{a} \frac{\Re\left(\psi_{s}\left(\frac{a^{\prime}}{a} s, \varphi(x)\right)\right)}{\Im\left(\psi\left(\frac{a^{\prime}}{a} s, \varphi(x)\right)\right)},
\end{aligned}
$$


and $\varphi$ satisfying (11) is a contact transform satisfying $g_{\varphi} \circ \Pi \circ \Psi_{\phi}=\Pi \circ \Psi_{\psi} \circ(S, X, \Theta)$. Moreover, such a function $h$ exists: using the fact that $\varphi$ satisfies (11), one may verify that

$$
\begin{aligned}
\frac{\partial}{\partial s}\left(\frac{\Im\left(\psi_{s}\left(\frac{a^{\prime}}{a} s, \varphi(x)\right)\right)}{\Im\left(\psi\left(\frac{a^{\prime}}{a} s, \varphi(x)\right)\right)} \dot{\varphi}(x)\right. & \left.-\frac{\Im\left(\phi_{s}(s, x)\right)}{\Im(\phi(s, x))}\right) \\
& =\frac{\partial}{\partial x}\left(\frac{\Re\left(\phi_{s}(s, x)\right)}{\Im(\phi(s, x))}-\frac{a^{\prime}}{a} \frac{\Re\left(\psi_{s}\left(\frac{a^{\prime}}{a} s, \varphi(x)\right)\right)}{\Im\left(\psi\left(\frac{a^{\prime}}{a} s, \varphi(x)\right)\right)}\right)
\end{aligned}
$$

and so, the system of equations for $h$ has a solution by the Poincaré lemma.

Finally, let us take a map $(s, x, \theta) \longmapsto\left(\frac{a^{\prime}}{a} s, \varphi(x), \theta+h(s, x)\right)$ with $\varphi$ satisfying (11), $\varphi(0)=0, \varphi(b)=b^{\prime}, \dot{\varphi} \geq \frac{a^{\prime}}{a}$ and $h$ satisfying (12) and (13). Then, it induces a quasiconformal lift of $g_{\varphi}, g: \widetilde{\Omega}_{a, b} \backslash\{z=0\} \longrightarrow \widetilde{\Omega}_{a^{\prime}, b^{\prime}} \backslash\{z=0\}$. Assume moreover that $g$ can be extended continuously to a map in $\mathcal{F}_{\phi, \psi}$. Then, Corollary 1.13 ensures that

$$
M\left(g\left(\widetilde{\Gamma}_{\phi}\right)\right)=\int_{\widetilde{\Omega}_{a, b}} K(., g)^{2}\left(\Pi^{*} \rho_{\phi}\right)^{4} \mathrm{~d} L^{3} .
$$

Now, let $\widetilde{\Gamma}$ be the family of all horizontal curves in $\widetilde{\Omega}_{a, b}$ connecting $\partial \widetilde{\Omega}_{0}$ and $\partial \widetilde{\Omega}_{a}$. Then, $\Pi^{*} \rho_{\phi} \in \operatorname{adm}(\widetilde{\Gamma})$ (see Lemma $\underline{3.14}$ ). Moreover, by definition of $\mathcal{F}_{\phi, \psi}$, for every $f \in \mathcal{F}_{\phi, \psi}, g\left(\widetilde{\Gamma}_{\phi}\right) \subset f(\widetilde{\Gamma})$ so that

$$
M\left(g\left(\widetilde{\Gamma}_{\phi}\right)\right) \leq M(f(\widetilde{\Gamma}))
$$

for every $f \in \mathcal{F}_{\phi, \psi}$. Thus, using Proposition [1.6. $g$ minimizes the mean distortion in $\mathcal{F}_{\phi, \psi}$ for the density $\Pi^{*} \rho_{\phi}$. So, we just showed the following.

Theorem 3.8. Let $_{g_{\varphi}}: \Omega_{a, b} \longrightarrow \Omega_{a^{\prime}, b^{\prime}}$ be a minimizer of the mean distortion in $\mathcal{G}_{\phi, \psi}$ for the density $\rho_{\phi}$ with $\varphi$ satisfying (11). Then, there is a quasiconformal lift of $g_{\varphi}, g: \widetilde{\Omega}_{a, b} \backslash\{z=0\} \longrightarrow \widetilde{\Omega}_{a^{\prime}, b^{\prime}} \backslash\{z=0\}$. If $g$ can be extended continuously to $a$ map in $\mathcal{F}_{\phi, \psi}$, then $g$ minimizes the mean distortion in $\mathcal{F}_{\phi, \psi}$ for the density $\Pi^{*} \rho_{\phi}$.

To end this section, we give a general theorem for lifting quasiconformal mappings from $\mathbb{H}$. It produces a new way to lift quasiconformal mappings from the plane to the Heisenberg group different from [CT95, BHIT06]. The formulation of the theorem (to be precise the link between the formula $r^{4} \frac{\mathrm{Jac} g}{(\Im(g))^{2}}=1$ and the preservation of the hyperbolic area form) is heavily inspired by a work in progress by Cano and Platis (see ACIDP16]). In their work, the authors create a setting in which the lifting theorem fits. The proof they give is therefore more conceptual than the one we give here (in the appendix) which relies on the same kind of computations as the proof of Proposition 3.7

Theorem 3.9. Let $g: \Omega \longrightarrow \Omega^{\prime}$ be a $C^{2}$-quasiconformal map between simply connected domains of $\mathbb{H}$. Let $\widetilde{\Omega}=\Pi^{-1}(\Omega)$ and $\widetilde{\Omega}^{\prime}=\Pi^{-1}\left(\Omega^{\prime}\right)$.

- If $g$ is a symplectomorphism with respect to the hyperbolic area form of $\mathbb{H}$ (i.e., for every $w \in \mathbb{H}$, $\frac{\operatorname{Jac} g(w)}{(\Im(g(w)))^{2}}=\frac{1}{(\Im(w))^{2}}$ where Jac $g$ is the Jacobian determinant of $g$ ), then there is a quasiconformal map $f: \widetilde{\Omega} \longrightarrow \widetilde{\Omega}^{\prime}$ such 
that $\Pi \circ f=g \circ \Pi$. Moreover, $f$ is of the form:

$$
(z, t) \longmapsto\left(z\left(\operatorname{Jac} g\left(t+i|z|^{2}\right)\right)^{\frac{1}{4}} e^{i h\left(t+i|z|^{2}\right)}, \Re\left(g\left(t+i|z|^{2}\right)\right)\right),
$$

where $h: \Omega \longrightarrow \mathbb{R}$ is given by the following equations, for every $w \in \Omega$ :

$$
\begin{aligned}
& \frac{\partial h}{\partial w}(w)=\frac{1}{4 \Im(w)}-\frac{1}{2 \Im(g(w))} \frac{\partial \Re(g)}{\partial w}(w), \\
& \frac{\partial h}{\partial \bar{w}}(w)=\frac{1}{4 \Im(w)}-\frac{1}{2 \Im(g(w))} \frac{\partial \Re(g)}{\partial \bar{w}}(w) .
\end{aligned}
$$

- Conversely, if $g$ can be lifted by $\Pi$ into a quasiconformal map between $\widetilde{\Omega}$ and $\widetilde{\Omega}^{\prime}$, then $g$ is a symplectomorphism with respect to the hyperbolic area form of $\mathbb{H}$ and $f$ has the form given previously.

Remark 3.10. Proposition 3.7 is a version of Theorem 3.9 in the case

$$
g=g_{\varphi}=\psi \circ f_{\varphi} \circ \phi^{-1} .
$$

In particular, using holomorphicity of $\phi$ and $\psi$, and $\operatorname{Jac} f_{\varphi}=\frac{a^{\prime}}{a} \dot{\varphi}$, condition (11) is equivalent to

$$
\frac{\operatorname{Jac}\left(g_{\varphi}\right)(\phi(s, x))}{\left(\Im\left(g_{\varphi}(\phi(s, x))\right)\right)^{2}}=\frac{a^{\prime}}{a} \dot{\varphi}(x) \frac{\left|\psi^{\prime}\left(f_{\varphi}(s, x)\right)\right|^{2}}{\left|\phi^{\prime}(s, x)\right|^{2}\left(\Im\left(\psi\left(f_{\varphi}(s, x)\right)\right)\right)^{2}}=\frac{1}{(\Im(\phi(s, x)))^{2}}
$$

so that condition (11) is equivalent to $g_{\varphi}$ being symplectic with respect to the hyperbolic area form.

3.3. Geometric conditions for uniqueness of the construction. In this section, we will prove that when there is a minimizer of the mean distortion in $\mathcal{G}_{\phi, \psi}$ for the density $\rho_{\phi}$ that can be lifted into a quasiconformal map in $\mathcal{F}_{\phi, \psi}$, then every minimizer of the mean distortion in $\mathcal{F}_{\phi, \psi}$ for the density $\Pi^{*} \rho_{\phi}$ is constructed by that process. The section is dedicated to the proof of the following theorem, which may be understood as a converse of Proposition 1.12 in the case of domains $\Omega$ and $\Omega^{\prime}$ biholomorphic to rectangles plus boundary conditions.

Theorem 3.11. Let $f: \widetilde{\Omega}_{a, b} \longrightarrow \widetilde{\Omega}_{a^{\prime}, b^{\prime}}$ be a quasiconformal map in $\mathcal{F}_{\phi, \psi}$ such that $f_{*} \Pi^{*} \rho_{\phi}=\Pi^{*} \rho_{\psi}$. Suppose that $\Pi^{*} \rho_{\phi}$ (resp., $\left.\Pi^{*} \rho_{\psi}\right)$ is extremal for $\widetilde{\Gamma}_{\phi}$ (resp., $\left.\widetilde{\Gamma}_{\psi}\right)$. Then, $f$ is a lift of a minimizer of the mean distortion in $\mathcal{G}_{\phi, \psi}$ for the density $\rho_{\phi}$. Namely, there is a quasiconformal map $g \in \mathcal{G}_{\phi, \psi}$ such that $\Pi \circ f=g \circ \Pi$ and $g_{*} \rho_{\phi}=\rho_{\psi}$.

Remark 3.12. It could be tempting to phrase the previous theorem using modulus of a curve family as: if $\Pi^{*} \rho_{\phi}$ (resp., $\left.\Pi^{*} \rho_{\psi}\right)$ is extremal for $\widetilde{\Gamma}_{\phi}\left(\right.$ resp., $\left.\widetilde{\Gamma}_{\psi}\right)$, then every quasiconformal map $f \in \mathcal{F}_{\phi, \psi}$ such that

$$
M\left(f\left(\widetilde{\Gamma}_{\phi}\right)\right)=\int_{\widetilde{\Omega}_{a, b}} K(., f)^{2}\left(\Pi^{*} \rho_{\phi}\right)^{4} \mathrm{~d} L^{3}
$$

is a lift of a $g_{\varphi}$.

But this is not what Theorem 3.11 states. In fact, it concerns maps $f \in \mathcal{F}_{\phi, \psi}$ satisfying

$$
M\left(\widetilde{\Gamma}_{\psi}\right)=\int_{\widetilde{\Omega}_{a, b}} K(., f)^{2}\left(\Pi^{*} \rho_{\phi}\right)^{4} \mathrm{~d} L^{3} .
$$

Nevertheless, we can still phrase Theorem 3.11 using moduli of curve families - only not with $f\left(\widetilde{\Gamma}_{\phi}\right)$. 
Let $\widetilde{\Gamma}$ be the family of all horizontal curves in $\widetilde{\Omega}_{a, b}$ connecting $\partial \widetilde{\Omega}_{0}$ and $\partial \widetilde{\Omega}_{a}$. Then, $\Pi^{*} \rho_{\phi} \in \operatorname{adm}(\widetilde{\Gamma})$ (see Lemma 13.14 ) and so it is extremal for $\widetilde{\Gamma}$. In the same way, $\Pi^{*} \rho_{\psi} \in \operatorname{adm}\left(\widetilde{\Gamma}^{\prime}\right)$ is also extremal where $\widetilde{\Gamma}^{\prime}$ is the family of all horizontal curves in $\widetilde{\Omega}_{a^{\prime}, b^{\prime}}$ connecting $\partial \widetilde{\Omega}_{0}^{\prime}$ and $\partial \widetilde{\Omega}_{a^{\prime}}^{\prime}$. Now, by definition of $\mathcal{F}_{\phi, \psi}$, for every $f \in \mathcal{F}_{\phi, \psi}$, $f(\widetilde{\Gamma})=\widetilde{\Gamma}^{\prime}$. So,

$$
f_{*} \Pi^{*} \rho_{\phi}=\Pi^{*} \rho_{\psi} \Longleftrightarrow M(f(\widetilde{\Gamma}))=\int_{\widetilde{\Omega}_{a, b}} K(., f)^{2}\left(\Pi^{*} \rho_{\phi}\right)^{4} \mathrm{~d} L^{3} .
$$

So, Theorem 3.11 is equivalent to the following theorem.

Theorem 3.13. Let $\widetilde{\Gamma}$ be the family of all horizontal curves in $\widetilde{\Omega}_{a, b}$ connecting $\partial \widetilde{\Omega}_{0}$ and $\partial \widetilde{\Omega}_{a}$, and let $\widetilde{\Gamma}^{\prime}$ be the family of all horizontal curves in $\widetilde{\Omega}_{a^{\prime}, b^{\prime}}$ connecting $\partial \widetilde{\Omega}_{0}^{\prime}$ and $\partial \widetilde{\Omega}_{a^{\prime}}^{\prime}$. Assume that $\Pi^{*} \rho_{\phi}\left(\right.$ resp., $\left.\Pi^{*} \rho_{\psi}\right)$ is extremal for $\widetilde{\Gamma}\left(\right.$ resp.,$\left.\widetilde{\Gamma}^{\prime}\right)$. Let $f \in \mathcal{F}_{\phi, \psi}$ be a quasiconformal map satisfying

$$
M(f(\widetilde{\Gamma}))=\int_{\widetilde{\Omega}_{a, b}} K(., f)^{2}\left(\Pi^{*} \rho_{\phi}\right)^{4} \mathrm{~d} L^{3} .
$$

Then, $f$ is a lift of one of the $g_{\varphi}$.

The proof of Theorem 3.11 follows the same steps as the one of Theorem 2.5. Again the main thing we have to prove is that a quasiconformal map $f=\left(f_{1}, f_{2}\right)$ as in the previous theorem has the property: $\left(f_{2}+i\left|f_{1}\right|^{2}\right)(z, t)$ does not depend on $\arg (z)$. Let us set a quasiconformal map $f: \widetilde{\Omega}_{a, b} \longrightarrow \widetilde{\Omega}_{a^{\prime}, b^{\prime}}$ with the hypothesis of the theorem. The first objective is to show that a map $f \in \mathcal{F}_{\phi, \psi}$ such that $f_{*} \Pi^{*} \rho_{\phi}=\Pi^{*} \rho_{\psi}$ must be defined by a map $(S, X, \Theta)$ that sends a curve

$$
(s, x, \alpha+\tau(s, x)) \text { with } \tau_{s}(s, x)=-\frac{\Re\left(\phi_{s}(s, x)\right)}{2 \Im(\phi(s, x))}
$$

on a curve

$$
\left(\frac{a^{\prime}}{a} s, x^{\prime}, \alpha^{\prime}+v\left(\frac{a^{\prime}}{a} s, x^{\prime}\right)\right) \text { with } v_{s}(s, x)=-\frac{\Re\left(\psi_{s}(s, x)\right)}{2 \Im(\psi(s, x))} .
$$

For that, we will follow essentially what we did in Section 2.2. First, a curve $\widetilde{\gamma}(t)=(s(t), x(t), \theta(t))$ in $] 0, a[\times] 0, b[\times \mathbb{R}$ (resp., in $] 0, a^{\prime}[\times] 0, b^{\prime}[\times \mathbb{R})$ is said to be horizontal if $\Psi_{\phi}(\widetilde{\gamma}(t))$ is horizontal in $\widetilde{\Omega}_{a, b}$ (resp., $\Psi_{\psi}(\widetilde{\gamma}(t))$ is horizontal in $\widetilde{\Omega}_{a^{\prime}, b^{\prime}}$ ).

Lemma 3.14. Let $\widetilde{\Gamma}$ be the family of all horizontal curves

$$
\widetilde{\gamma}(t)=(s(t), x(t), \theta(t)) \text { such that } s(0)=0 \text { and } s(a)=a^{\prime} .
$$

Then, for every $\widetilde{\gamma} \in \widetilde{\Gamma}$,

$$
\int_{\Psi_{\phi}(\widetilde{\gamma})} \Pi^{*} \rho_{\phi} \mathrm{d} l \geq 1
$$

Moreover, we have equality if and only if $\Psi_{\phi}(\widetilde{\gamma}) \in \widetilde{\Gamma}_{\phi}$. 
Proof. Let $\widetilde{\gamma}$ be a curve in $\widetilde{\Gamma}$ and $\gamma=\Psi_{\phi}(\widetilde{\gamma})$. Then, we have the following:

$$
\begin{aligned}
\int_{\gamma} \Pi^{*} \rho_{\phi} \mathrm{d} l & =\int_{0}^{a} \frac{2\left|\gamma_{1}(t)\right|\left|\dot{\gamma}_{1}(t)\right|}{a\left|\phi^{\prime}\left(\phi^{-1}(\Pi \circ \gamma)(t)\right)\right|} \mathrm{d} t \\
& =\frac{1}{a} \int_{0}^{a} \frac{(\Pi \circ \gamma)(t)}{\left|\phi^{\prime}\left(\phi^{-1}(\Pi \circ \gamma)(t)\right)\right|} \mathrm{d} t \\
& =\frac{1}{a} \int_{0}^{a}|\dot{s}(t)+i \dot{x}(t)| \mathrm{d} t \\
& \geq \frac{1}{a} \int_{0}^{a}|\dot{s}(t)| \mathrm{d} t \\
& =1 .
\end{aligned}
$$

We have equality here if and only if $\dot{x}=0$. One may then verify that a curve $\left(s(t), x_{0}, \theta(t)\right)$ is horizontal if and only if its image by $\Psi_{\phi}$ is an element of $\widetilde{\Gamma}_{\phi}$.

Now, we may prove the following the same way that we proved Proposition 2.7

Proposition 3.15. In our setting we have

$$
f\left(\widetilde{\Gamma}_{\phi}\right)=\widetilde{\Gamma}_{\psi} .
$$

Moreover, $f$ maps (potential pieces of) the vertical axis to itself.

Thus, according to this proposition, we can restrict our attention to $\widetilde{\Omega}_{a, b} \backslash\{z=$ $0\}$ and $\widetilde{\Omega}_{a^{\prime}, b^{\prime}} \backslash\{z=0\}$, meaning that $f$ induces a well-defined map $(S, X, \Theta)$ : ] $0, a[\times] 0, b[\times \mathbb{R} \longrightarrow] 0, a^{\prime}[\times] 0, b^{\prime}[\times \mathbb{R}$ that sends a curve

$$
\widetilde{\gamma}_{(x, \alpha)}=(s, x, \alpha+\tau(s, x)) \text { with } \dot{\tau}(s, x)=-\frac{\Re\left(\phi_{s}(s, x)\right)}{2 \Im(\phi(s, x))}
$$

on a curve

$$
\widetilde{\delta}_{\left(x^{\prime}, \alpha^{\prime}\right)}(s)=\left(\zeta_{(x, \alpha)}(s), x^{\prime}, \alpha^{\prime}+v\left(\zeta_{(x, \alpha)}(s), x^{\prime}\right)\right) \text { with } \dot{v}(s, x)=-\frac{\Re\left(\psi_{s}(s, x)\right)}{2 \Im(\psi(s, x))} .
$$

It remains to show that

$$
\zeta_{(x, \alpha)}(s)=\frac{a^{\prime}}{a} s .
$$

Proposition 3.16. The map $(S, X, \theta)$ sends a curve $\widetilde{\gamma}_{(x, \alpha)}(s)=(s, x, \alpha+\tau(s, x))$ with $\dot{\tau}(s, x)=-\frac{\Re\left(\phi_{s}(s, x)\right)}{2 \Im(\phi(s, x))}$ on a curve $\widetilde{\delta}_{\left(x^{\prime}, \alpha^{\prime}\right)}(s)=\left(\frac{a^{\prime}}{a} s, x^{\prime}, \alpha^{\prime}+v\left(\frac{a^{\prime}}{a} s, x^{\prime}\right)\right)$ with $\dot{v}(s, x)=-\frac{\Re\left(\psi_{s}(s, x)\right)}{2 \Im(\psi(s, x))}$.

Proof. Again, the proof is very similar to the one of Proposition 2.8, We consider the following two complex vector fields:

$$
U:=-\frac{i z \phi^{\prime}\left(\phi^{-1}\left(t+i|z|^{2}\right)\right)}{2|z|^{2}} Z \quad \text { and } \quad \bar{U}:=\frac{i \bar{z} \bar{\phi}^{\prime}\left(\phi^{-1}\left(t+i|z|^{2}\right)\right)}{2|z|^{2}} \bar{Z} .
$$

Then, using the same method as in Proposition 2.8, one may check that

$$
|U(\Pi \circ f)|-|\bar{U}(\Pi \circ f)|=\frac{\left|f_{1}\right|\left|\phi^{\prime}\left(\phi^{-1}\left(t+i|z|^{2}\right)\right)\right|}{|z|}\left(\left|Z f_{1}\right|-\left|\bar{Z} f_{1}\right|\right) .
$$

Now, since

$$
f_{*} \widetilde{\rho}_{\phi} \circ f=\widetilde{\rho}_{\psi} \circ f \text { with } \widetilde{\rho}_{\phi}=\Pi^{*} \rho_{\phi}=\Pi^{*} \phi_{*} \rho_{0} \text { and } \widetilde{\rho}_{\psi}=\Pi^{*} \rho_{\psi}=\Pi^{*} \psi_{*} \rho_{0}^{\prime}
$$


we have

$$
f_{*} \widetilde{\rho}_{\phi} \circ f=\frac{2\left|f_{1}\right|}{a(|U(\Pi \circ f)|-|\bar{U}(\Pi \circ f)|)}=\frac{2\left|f_{1}\right|}{a^{\prime}\left|\psi^{\prime}\left(\psi^{-1} \circ \Pi \circ f\right)\right|} .
$$

This leads to

$$
|U(\Pi \circ f)|-|\bar{U}(\Pi \circ f)|=\frac{a^{\prime}}{a}\left|\psi^{\prime}\left(\psi^{-1} \circ \Pi \circ f\right)\right| .
$$

Now, let $\widetilde{\gamma}_{(x, \alpha)}(s)=(s, x, \alpha+\tau(s, x))$ with $\dot{\tau}(s, x)=-\frac{\Re\left(\phi_{s}(s, x)\right)}{2 \Im(\phi(s, x))}$ and $\gamma_{(x, \alpha)}(s)$ $=\Psi_{\phi}\left(\widetilde{\gamma}_{(x, \alpha)}(s)\right)$. Then we have,

$$
\Pi \circ f \circ \gamma_{(x, \alpha)}(s)=\psi\left(\zeta_{(x, \alpha)}(s), x^{\prime}\right) .
$$

Thus,

But we also have

$$
\left|\frac{\mathrm{d}}{\mathrm{d} s}\left(\left(\Pi \circ f \circ \gamma_{(x, \alpha)}\right)\right)(s)\right|=\left|\psi^{\prime}\left(\zeta_{(x, \alpha)}(s), x^{\prime}\right)\right|\left|\dot{\zeta}_{(x, \alpha)}(s)\right| .
$$

$$
\begin{aligned}
\left|\frac{\mathrm{d}}{\mathrm{d} s}\left(\left(\Pi \circ f \circ \gamma_{(x, \alpha)}\right)\right)(s)\right| & =\left|U(\Pi \circ f)\left(\gamma_{(x, \alpha)}(s)\right)\right|-\left|\bar{U}(\Pi \circ f)\left(\gamma_{(x, \alpha)}(s)\right)\right| \\
& =\frac{a^{\prime}}{a}\left|\psi^{\prime}\left(\psi^{-1} \circ \Pi \circ f \circ \gamma_{(x, \alpha)}\right)(s)\right| \\
& =\frac{a^{\prime}}{a}\left|\psi^{\prime}\left(\zeta_{(x, \alpha)}(s), x^{\prime}\right)\right| .
\end{aligned}
$$

So, $\zeta_{(x, \alpha)}(s)=\frac{a^{\prime}}{a} s$ which ends the proof.

Finally, a rather long but elementary calculus (done in the appendix) shows the following.

Proposition 3.17. The map $(S, X, \Theta)$ satisfies $X_{\theta}=0$.

Combining Propositions 3.16 and 3.17 together with Corollary 1.13 is enough to prove Theorem 3.11. Before giving examples, we sum up the results gotten in Section 3. In the following theorem, we denote by $\widetilde{\Gamma}$ the family of all horizontal curves in $\widetilde{\Omega}_{a, b}$ connecting $\partial \widetilde{\Omega}_{0}$ and $\partial \widetilde{\Omega}_{a}$.

Theorem 3.18. If $\Pi^{*} \rho_{\phi}$ is extremal for $\widetilde{\Gamma}_{\phi}$ and $\Pi^{*} \rho_{\psi}$ is extremal for $\widetilde{\Gamma}_{\psi}$, then we have the dichotomy:

1) If there is a minimizer $g$ of the mean distortion in $\mathcal{G}_{\phi, \psi}$ for the density $\rho_{\phi}$ that can be lifted into a quasiconformal map $f \in \mathcal{F}_{\phi, \psi}$, then $f$ minimizes the mean distortion in $\mathcal{F}_{\phi, \psi}$ for the density $\Pi^{*} \rho_{\phi}$. Moreover, every minimizer of the mean distortion in $\mathcal{F}_{\phi, \psi}$ for the density $\Pi^{*} \rho_{\phi}$ is a lift of a minimizer of the mean distortion in $\mathcal{G}_{\phi, \psi}$ for the density $\rho_{\phi}$.

2) If none of the minimizers of the mean distortion in $\mathcal{G}_{\phi, \psi}$ for the density $\rho_{\phi}$ can be lifted into a quasiconformal map in $\mathcal{F}_{\phi, \psi}$, then, for every $f \in \mathcal{F}_{\phi, \psi}$,

$$
M(f(\widetilde{\Gamma}))<\int_{\widetilde{\Omega}_{a, b}} K(., f)^{2}\left(\Pi^{*} \rho_{\phi}\right)^{4} \mathrm{~d} L^{3} .
$$

Proof. We only have one thing left to prove here: in the first case, every minimizer of the mean distortion in $\mathcal{F}_{\phi, \psi}$ for the density $\Pi^{*} \rho_{\phi}$ is a lift of a minimizer of the mean distortion in $\mathcal{G}_{\phi, \psi}$ for the density $\rho_{\phi}$. We will show that in that case, a minimizer $f$ must satisfy $f_{*} \Pi^{*} \rho_{\phi}=\Pi^{*} \rho_{\psi}$. Then, the result will follow by Theorem 3.11. So, pick $f \in \mathcal{F}_{\phi, \psi}$ as a minimizer of the mean distortion and denote by $f_{0}$ 
the minimizer of the mean distortion coming from the hypothesis. Then, it is clear that $f_{0}\left(\widetilde{\Gamma}_{\phi}\right)=\widetilde{\Gamma}_{\psi}$. Moreover, Corollary 1.13 ensures that

$$
M\left(f_{0}\left(\widetilde{\Gamma}_{\phi}\right)\right)=\int_{\widetilde{\Omega}_{a, b}} K\left(., f_{0}\right)^{2}\left(\Pi^{*} \rho_{\phi}\right)^{4} \mathrm{~d} L^{3}
$$

So,

$$
M\left(\widetilde{\Gamma}_{\psi}\right)=\int_{\widetilde{\Omega}_{a, b}} K\left(., f_{0}\right)^{2}\left(\Pi^{*} \rho_{\phi}\right)^{4} \mathrm{~d} L^{3}=\int_{\widetilde{\Omega}_{a, b}} K(., f)^{2}\left(\Pi^{*} \rho_{\phi}\right)^{4} \mathrm{~d} L^{3},
$$

which means that $f_{*} \Pi^{*} \rho_{\phi}$ is extremal for $\widetilde{\Gamma}_{\psi}$. Since $\Pi^{*} \rho_{\psi}$ and $f_{*} \Pi^{*} \rho_{\phi}$ are continuous, they are equal by Lemma 1.3 .

We now wish to give two examples of the construction. The first one is between spherical annuli on the Heisenberg group and comes from [BFP13, BFP15 where extremality and uniqueness were proved. Here, it is constructed using the holomorphic map $z \longmapsto e^{z}$. Applying Proposition 3.7 and Theorem 3.11, we are able to reconstruct the map and prove its uniqueness. The second example uses the translation $z \longmapsto z+i$.

Example 3.19. Let us consider two half-annuli in $\mathbb{H}$ :

$$
A_{a}:=\left\{w \in \mathbb{H}|1<| w \mid<a^{2}\right\} \quad \text { and } \quad A_{a^{k}}:=\left\{w \in \mathbb{H}|1<| w \mid<a^{2 k}\right\}
$$

for $k<1$ and $a>1$. Then,

$$
A_{a}=\phi(] 0,2 \ln (a)[\times] 0, \pi[) \quad \text { and } \quad A_{a^{k}}=\psi(] 0,2 k \ln (a)[\times] 0, \pi[),
$$

where $\phi(s, x)=\psi(s, x)=e^{s+i x}$. Then,

$$
\frac{\left|\phi^{\prime}(s, x)\right|^{2}}{\Im(\phi(s, x))^{2}}=\frac{1}{\sin ^{2}(x)}
$$

is a function of $x$ only. Moreover, we denote

$$
\widetilde{A}_{a}=\overline{\Pi^{-1}\left(A_{a}\right)}=\left\{(z, t) \in \mathbf{H} \mid 1<\|(z, t)\|_{\mathbf{H}}<a\right\}
$$

and

$$
\widetilde{A}_{a^{k}}=\overline{\Pi^{-1}\left(A_{a^{k}}\right)}=\left\{(z, t) \in \mathbf{H} \mid 1<\|(z, t)\|_{\mathbf{H}}<a^{k}\right\}
$$

the spherical annuli in $\mathbf{H}$. The set $\mathcal{F}_{\phi, \psi}$ here is the set of quasiconformal maps $f: \widetilde{A}_{a} \longrightarrow \widetilde{A}_{a^{k}}$ that extend homeomorphically on $\left\{(z, t) \in \mathbf{H} \mid 1 \leq\|(z, t)\|_{\mathbf{H}} \leq a\right\}$, sending $\left\{\|(z, t)\|_{\mathbf{H}}=1\right\}$ on $\left\{\|(z, t)\|_{\mathbf{H}}=1\right\}$ and $\left\{\|(z, t)\|_{\mathbf{H}}=a\right\}$ on $\left\{\|(z, t)\|_{\mathbf{H}}=\right.$ $\left.a^{k}\right\}$. Finally, the family of curves considered here is the family of radial curves

$$
\gamma_{(x, \alpha)}(s)=\left(\sqrt{e^{s} \sin x} e^{i\left(\alpha-\frac{\cot x}{2} s\right)}, e^{s} \cos x\right)
$$

which has modulus $\pi^{2} \ln (a)^{-3}$ with extremal density

$$
\rho_{\phi}(z, t)=\frac{|z|}{\ln (a) \sqrt{t^{2}+|z|^{4}}}
$$

for $\widetilde{A}_{a}$ and $\pi^{2} \ln \left(a^{k}\right)^{-3}$ with extremal density

$$
\rho_{\psi}(z, t)=\frac{|z|}{\ln \left(a^{k}\right) \sqrt{t^{2}+|z|^{4}}}
$$

for $\widetilde{A}_{a^{k}}$ (see Figure 2) 


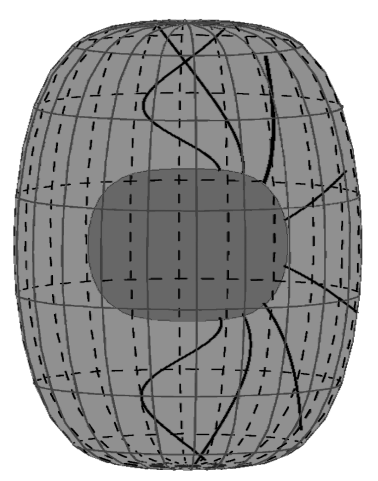

Figure 2. Spherical annulus foliated by radial curves (foliation given by rotations around the vertical axis of drawn curves and the two pieces of the vertical axis itself).

According to Proposition 3.7, if a lift $(S, X, \Theta)$ of $g_{\varphi}$ exists, it must verify the following:

$$
\begin{gathered}
S(s, x, \theta)=k s, X(s, x, \theta)=\varphi(x), \\
\dot{\varphi}(x) \frac{\sin ^{2}(x)}{\sin ^{2}(\varphi(x))}=k^{-1} .
\end{gathered}
$$

Thus, solving the ordinary differential equation, we find for every $x$,

$$
\varphi(x)=\cot ^{-1}\left(k^{-1} \cot (x)+D\right) \text { where } D \in \mathbb{R} .
$$

Moreover, $\dot{\varphi}(x) \geq k$ for every $x$. For $\varphi(x)=\cot ^{-1}\left(k^{-1} \cot (x)+D\right)$, this is equivalent to

$$
k+2 D \cot (x)+k D^{2} \leq 1 \text { for every } x,
$$

which is possible if and only if $D=0$. So,

$$
\varphi(x)=\cot ^{-1}\left(k^{-1} \cot (x)\right)
$$

for every $x \in] 0, \pi[$. In particular, notice that $\varphi$ extends continuously in a homeomorphism from $[0, \pi]$ to $[0, \pi]$. By Proposition 3.7 again, we know that we can find the function $\Theta$ to make $(S, X, \Theta)$ define a quasiconformal map between spherical annuli (minus pieces of the vertical axis):

$$
\Theta(s, x, \theta)=\theta+h(s, x),
$$

where $h$ verifies

$$
2 h_{s}(s, x)=0 \text { and } 2 h_{x}(s, x)=\dot{\varphi}(x)-1 .
$$

Thus, we find

$$
h(s, x)=\frac{\varphi(x)-x}{2}+\theta_{0} \text { with } \theta_{0} \in \mathbb{R} .
$$


Using $\Psi_{\phi}$ one is invited to check that in usual coordinates, it gives (up to composition with a rotation around the $t$-axis) the map

$$
\begin{aligned}
f: \widetilde{A}_{a} \backslash\{z=0\} & \longrightarrow \\
(z, t) & \longmapsto\left(\left.\left.\sqrt{k} z\left(\frac{t-i|z|^{2}}{t-i k|z|^{2}}\right)^{\frac{1}{2}}|t+i| z\right|^{2}\right|^{\frac{k-1}{2}}, t \frac{\left.\left.|t+i| z\right|^{2}\right|^{k}}{\left.|t+i k| z\right|^{2} \mid}\right) .
\end{aligned}
$$

Then, $f$ extends in a homeomorphism in $\mathcal{F}_{\phi, \psi}$. This is the map studied in BFP13. called the radial stretch map. By Theorem 3.8, $f$ minimizes the mean distortion in $\mathcal{F}_{\phi}$ for the density $\widetilde{\rho}_{\phi}$. Finally, the first part of Theorem 3.18 ensures that, up to composition with vertical rotations, $f$ is the only minimizer of the mean distortion in $\mathcal{F}_{\phi, \psi}$ for the density $\rho_{\phi}$.

The radial stretch map appeared in various contexts, for instance, in Min94, Aus16.

Example 3.20. Let us consider a subset of a cylinder

$$
D_{r, R}:=\left\{\left.(z, t) \in \mathbf{H}|0<t<r, 1<| z\right|^{2}<R+1\right\} .
$$

We are interested in the same minimization problem as in Section 2 but this time between $D_{a, b}$ and $D_{a^{\prime}, b^{\prime}}$ with $\frac{a\left(b^{\prime}+1\right)}{a^{\prime}(b+1)}>1$. This means that we consider the foliation of $D_{a, b}$ given by the subset of $\widetilde{\Gamma}_{0}$ of curves that lie in $D_{a, b}$ (see Figure 3 ).

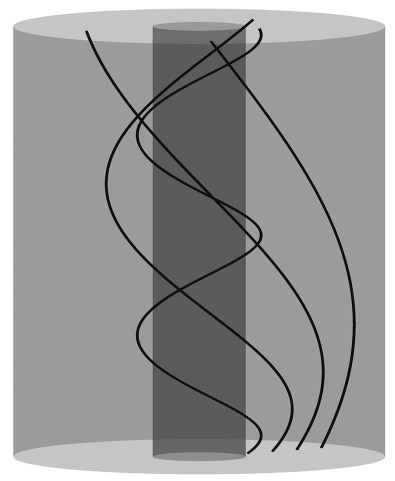

Figure 3. $D_{a, b}$ foliated by a subset of $\widetilde{\Gamma}_{0}$ (foliation given by rotations around the vertical axis of drawn curves).

Those cylinders are simply lifts up by $\Pi$ of rectangles $\phi\left(R_{a, b}\right)$ and $\phi\left(R_{a^{\prime}, b^{\prime}}\right)$ for $\phi(w)=w+i$. According to Theorem [3.9, a lift of one of the $g_{\varphi}$ exists only if $\phi\left(R_{a, b}\right)$ and $\phi\left(R_{a^{\prime}, b^{\prime}}\right)$ have the same (finite) hyperbolic area. Moreover, equation (11) writes as

$$
\frac{a^{\prime}}{a} \dot{\varphi}(x) \frac{(x+1)^{2}}{(\varphi(x)+1)^{2}}=1
$$


whose solutions are functions defined for $x$ by

$$
\varphi(x)=\frac{a^{\prime}}{a} \frac{x+1}{C x+C+1}-1 \text { with } C \in \mathbb{R} .
$$

From $\varphi(0)=0$, we deduce that

$$
C=\frac{a^{\prime}}{a}-1
$$

So, for every $x \in[0, b]$,

$$
\varphi(x)=\frac{a}{a^{\prime}} \frac{x}{\left(1-\frac{a}{a^{\prime}}\right) x+1} .
$$

Then, assume that $\phi\left(R_{a, b}\right)$ and $\phi\left(R_{a^{\prime}, b^{\prime}}\right)$ have the same hyperbolic area, meaning that,

$$
\frac{a b}{b+1}=\frac{a^{\prime} b^{\prime}}{b^{\prime}+1}
$$

Using $\frac{a}{a^{\prime}}=\frac{b^{\prime}(b+1)}{b\left(b^{\prime}+1\right)}$, we have then

$$
\varphi(b)=\frac{a}{a^{\prime}} \frac{b}{\left(1-\frac{a}{a^{\prime}}\right) b+1}=\frac{b^{\prime}(b+1)}{b\left(b^{\prime}+1\right)-b^{\prime}(b+1)+b^{\prime}+1}=b^{\prime} .
$$

So, $g_{\varphi}$ can be lifted to a contact map between $D_{a, b}$ and $D_{a^{\prime}, b^{\prime}}$. Moreover, for every $s \in[0, a], x \in[1, b+1]$,

$$
g_{\varphi}(s, x)=\frac{a^{\prime}}{a} s+i \frac{a^{\prime}}{a} \frac{x}{\left(\frac{a^{\prime}}{a}-1\right)(x-1)+\frac{a^{\prime}}{a}}=\frac{a^{\prime}}{a} s+i \frac{a^{\prime}}{a} \frac{x}{\left(\frac{a^{\prime}}{a}-1\right) x+1} .
$$

Replacing $\frac{a^{\prime}}{a}$ by $\frac{b\left(b^{\prime}+1\right)}{b^{\prime}(b+1)}$ in the imaginary part of $g_{\varphi}$, we find for every $s \in[0, a]$, $x \in[1, b]$,

$$
g_{\varphi}(s, x)=\frac{a^{\prime}}{a} s+i \frac{\left(b^{\prime}+1\right) x}{\left(1-\frac{b^{\prime}}{b}\right) x+\frac{b^{\prime}(b+1)}{b}}=\frac{a^{\prime}}{a} s+i \frac{\left(b^{\prime}+1\right) x}{\left(1-\frac{a\left(b^{\prime}+1\right)}{a^{\prime}(b+1)}\right) x+\frac{a\left(b^{\prime}+1\right)}{a^{\prime}}}
$$

which is exactly the restriction to $[1, b+1]$ of the function found in Section 2 from the rectangle $[0, a] \times[0, b+1]$ to the rectangle $\left[0, a^{\prime}\right] \times\left[0, b^{\prime}+1\right]$. So $g_{\varphi}$ can be lifted into a quasiconformal map between $D_{a, b}$ and $D_{a, b}$ and a lift is the restriction to $D_{a, b}$ of one of the $\tilde{f}_{\alpha}: C_{a, b+1} \longrightarrow C_{a^{\prime}, b^{\prime}+1}$ from Proposition 2.3. Finally, Theorem 3.8 ensures that lifts of $g_{\varphi}$ minimize the mean distortion in $\mathcal{F}_{\phi, \phi}$ for the density $\Pi^{*} \rho_{\phi}$. So, Theorem 3.18 gives the dichotomy:

- If $\phi\left(R_{a, b}\right)$ and $\phi\left(R_{a^{\prime}, b^{\prime}}\right)$ have the same hyperbolic area, then restrictions of $\tilde{f}_{\alpha}: C_{a, b+1} \longrightarrow C_{a^{\prime}, b^{\prime}+1}$ to $D_{a, b}$ minimize the mean distortion in $\mathcal{F}_{\phi, \phi}$ for the density $\Pi^{*} \rho_{\phi}$ and they are the only such minimizers.

- If $\phi\left(R_{a, b}\right)$ and $\phi\left(R_{a^{\prime}, b^{\prime}}\right)$ don't have the same hyperbolic area, then for every quasiconformal map $f \in \mathcal{F}_{\phi, \phi}$,

$$
M(f(\widetilde{\Gamma}))<\int_{D_{a, b}} K(., f)^{2}\left(\Pi^{*} \rho_{\phi}\right)^{4} \mathrm{~d} L^{3}
$$

where $\widetilde{\Gamma}$ is the family of horizontal curves in $D_{a, b}$ connecting the two boundary annuli. 


\section{APPENDIX}

Here, we provide some proofs left aside in the paper.

Proof of Lemma 2.2. First, it is a simple verification that maps $f_{\varphi}$ minimize the mean distortion in $\mathcal{G}$ for the density $\rho_{0}$. Moreover, since those maps satisfy

$$
M\left(\Gamma_{0}^{\prime}\right)=\int_{R_{a, b}} K\left(., f_{\varphi}\right) \rho_{0}^{2} \mathrm{~d} L^{2},
$$

every minimizer must also satisfy it. So, with the same argument as in Proposition 2.7. if $f \in \mathcal{G}$ minimizes the mean distortion functional, then $f\left(\Gamma_{0}\right)=\Gamma_{0}^{\prime}$. So for every $s, y$,

$$
f(s+i y)=\zeta_{y}(s)+i \varphi(y)
$$

for functions $\zeta_{y}$ and $\varphi$. Moreover, using a similar argument as the one in Proposition 2.8, we find that

$$
\zeta_{y}(s)=\frac{a^{\prime}}{a} s \text { for every } s, y
$$

To sum up,

$$
f(x+i y)=\frac{a^{\prime}}{a} x+i \varphi(y) \text { for every } x+i y \in R_{a, b} .
$$

For such a function to be orientation-preserving, $\dot{\varphi} \geq 0$ and, since $f$ sends horizontal boundary components on the corresponding ones, $\varphi(0)=0$ and $\varphi(b)=b^{\prime}$. Finally, the minimal stretching property in that case leads to $|\dot{\varphi}| \geq \frac{a^{\prime}}{a}$. So $f$ is one of the $f_{\varphi}$.

Proof of Theorem 3.9. Again, partial derivatives are denoted by an index.

For the first part, let $k: \Omega \longrightarrow \mathbb{C}$ be defined for every $w \in \Omega$ by

$$
k(w)=\frac{1}{4 \Im(w)}-\frac{(\Re(g))_{w}(w)}{2 \Im(g(w))} \quad \text { and } \quad \beta=k(w) \mathrm{d} w+\overline{k(w)} \mathrm{d} \bar{w} .
$$

Then, using the fact that $g$ is a symplectic map, a calculus shows that $\beta$ is closed, hence exact by the Poincaré lemma. So (since $\beta$ is a real 1 -form) there is a real valued function $h: \Omega \longrightarrow \mathbb{R}$ such that

$$
h_{w}=k \quad \text { and } \quad h_{\bar{w}}=\bar{k} .
$$

Now, it is a simple verification to check that the map $f$ defined in the theorem is a contact map and then, quasiconformality follows from Lemma 1.14.

For the second part, let $f: \widetilde{\Omega} \longrightarrow \widetilde{\Omega}^{\prime}$ be a contact lift of $g$. Here, it will be easier to compute in usual cylindrical coordinates $(r, \theta, t) \longmapsto\left(r e^{i \theta}, t\right) . f$ is then a map $(R, \Theta, T)$ with

$$
R(r, \theta, t)=\sqrt{\Im\left(g\left(t+i r^{2}\right)\right)} \quad \text { and } \quad T(r, \theta, t)=\Re\left(g\left(t+i r^{2}\right)\right) .
$$

The contact form $\omega$ is in those coordinates

$$
\omega=\mathrm{d} t+2 r^{2} \mathrm{~d} \theta .
$$

So, $f$ is contact if and only if the following three PDEs are satisfied:

$$
\begin{gathered}
T_{r}+2 R^{2} \Theta_{r}=0, \\
T_{t}+2 R^{2} \Theta_{t}=\lambda, \\
R^{2} \Theta_{\theta}=r^{2} \lambda,
\end{gathered}
$$


with $\lambda$ a nowhere vanishing function. Moreover, for every $(r, \theta, t)$,

$$
T_{r}(r, \theta, t)=2 i r\left((\Re(g))_{w}\left(t+i r^{2}\right)-(\Re(g))_{\bar{w}}\left(t+i r^{2}\right)\right)
$$

and

$$
T_{t}(r, \theta, t)=(\Re(g))_{w}\left(t+i r^{2}\right)+(\Re(g))_{\bar{w}}\left(t+i r^{2}\right) .
$$

So, we find

$$
\Theta_{r}(r, \theta, t)=\frac{i r}{\Im\left(g\left(t+i r^{2}\right)\right)}\left((\Re(g))_{\bar{w}}\left(t+i r^{2}\right)-\partial_{w}(\Re(g))\left(t+i r^{2}\right)\right)
$$

and deduce the following two:

$$
\Theta_{r, \theta}=0
$$

and

$$
\begin{aligned}
\Theta_{r, t}=\frac{-i r}{(\Im(g))^{2}}\left((\Im(g))_{w}+(\Im(g))_{\bar{w}}\right)\left((\Re(g))_{\bar{w}}\right. & \left.-(\Re(g))_{w}\right) \\
& +\frac{i r}{\Im(g)}\left((\Re(g))_{\bar{w}, \bar{w}}-(\Re(g))_{w, w}\right) .
\end{aligned}
$$

Moreover, we have

$$
\Theta_{\theta}=\frac{r^{2}}{\Im(g)}\left((\Re(g))_{w}+(\Re(g))_{\bar{w}}\right)+2 r^{2} \Theta_{t}
$$

and so, differentiating with respect to $r$, we find

$$
\Theta_{t}=\frac{r^{2} \operatorname{Jac} g}{2(\Im(g))^{2}}-\frac{(\Re(g))_{w}+(\Re(g))_{\bar{w}}}{2 \Im(g)} .
$$

From that, we deduce two things:

$$
\Theta_{\theta, t}=0 \text { and } \Theta_{\theta}=r^{4} \frac{\mathrm{Jac} g}{(\Im(g))^{2}} .
$$

So,

$$
\Theta_{\theta, \theta}=0 .
$$

Thus, $\Theta_{\theta}$ is constant with value 1 (if not so, $(R, \Theta, T)$ would not be a homeomorphism). It leads to

$$
\frac{\mathrm{Jac} g}{(\Im(g))^{2}}=\frac{1}{(\Im(w))^{2}}
$$

which ends the proof.

Proof of Proposition 3.17. The proof is similar to the one of Theorem 2.5. First, by hypothesis, for every $(s, x, \theta) \in] 0, a[\times] 0, b[\times \mathbb{R}$,

$$
S(s, x, \theta)=\frac{a^{\prime}}{a} s \quad \text { and } \quad \frac{\mathrm{d}}{\mathrm{d} s} X(s, x, \alpha+\tau(s, x))=0 .
$$

It leads to

$$
X_{s}=\frac{\Re\left(\phi_{s}\right)}{2 \Im(\phi)} X_{\theta}
$$


Now, since $(S, X, \Theta)$ defines a contact map, there is a nowhere vanishing function $\lambda$ such that

$$
\begin{aligned}
\frac{a^{\prime}}{a} \Re\left(\psi_{s}(S, X)\right)-\Im\left(\psi_{s}(S, X)\right) X_{s}+2 \Im(\psi(S, X)) \Theta_{s} & =\lambda \Re\left(\phi_{s}\right), \\
\Im\left(\psi_{s}(S, X)\right) X_{x}-2 \Im(\psi(S, X)) \Theta_{x} & =\lambda \Im\left(\phi_{s}\right), \\
-\Im\left(\psi_{s}(S, X)\right) X_{\theta}+2 \Im(\psi(S, X)) \Theta_{\theta} & =2 \lambda \Im(\phi) .
\end{aligned}
$$

From (25), since $\Im(\phi)>0$, we find

$$
\lambda=\frac{\Im(\psi(S, X))}{\Im(\phi)} \Theta_{\theta}-\frac{\Im\left(\psi_{s}(S, X)\right)}{2 \Im(\phi)} X_{\theta} .
$$

Replacing $\lambda$ by its value in (23) and (24), and using (22), we have the following:

$$
\begin{aligned}
\Theta_{s} & =\frac{\Re\left(\phi_{s}\right)}{2 \Im(\phi)} \Theta_{\theta}-\frac{a^{\prime}}{a} \frac{\Re\left(\psi_{s}(S, X)\right)}{2 \Im(\psi(S, X))}, \\
\Theta_{x} & =\frac{\Im\left(\psi_{s}(S, X)\right)}{2 \Im(\psi(S, X))} X_{x}+\frac{\Im\left(\phi_{s}\right)}{2 \Im(\phi)} \frac{\Im\left(\psi_{s}(S, X)\right)}{2 \Im(\psi(S, X))} X_{\theta}-\frac{\Im\left(\phi_{s}\right)}{2 \Im(\phi)} \Theta_{\theta} .
\end{aligned}
$$

From now, to follow computations, as a rule, we decide to express all partial derivatives of $\Re(\phi), \Re(\psi), \Im(\phi)$, and $\Im(\psi)$ with respect to $s$ (we do it using CauchyRiemann equations). Differentiating (26) and (27) with respect to $\theta$, we find

$$
\begin{aligned}
\Theta_{\theta, s}= & \frac{\Re\left(\phi_{s}\right)}{2 \Im(\phi)} \Theta_{\theta, \theta}-\frac{a^{\prime}}{a}\left(\frac{\Re\left(\psi_{s, x}(S, X)\right)}{2 \Im(\psi(S, X))}-\frac{\Re\left(\psi_{s}(S, X)\right) \Im\left(\psi_{x}(S, X)\right)}{2(\Im(\psi(S, X)))^{2}}\right) X_{\theta}, \\
\Theta_{\theta, x}= & \left.\frac{\Im\left(\psi_{s, x}(S, X)\right)}{2 \Im(\psi(S, X))}-\frac{\Im\left(\psi_{s}(S, X)\right) \Im\left(\psi_{x}(S, X)\right)}{2(\Im(\psi(S, X)))^{2}}\right) X_{\theta} X_{x} \\
& +\frac{\Im\left(\phi_{s}\right)}{2 \Im(\phi)}\left(\frac{\Im\left(\psi_{s, x}(S, X)\right)}{2 \Im(\psi(S, X))}-\frac{\Im\left(\psi_{s}(S, X)\right) \Im\left(\psi_{x}(S, X)\right)}{2(\Im(\psi(S, X)))^{2}}\right)\left(X_{\theta}\right)^{2} \\
& -\frac{\Im\left(\phi_{s}\right)}{2 \Im(\phi)} \Theta_{\theta, \theta}+\frac{\Im\left(\phi_{s}\right)}{2 \Im(\phi)} \frac{\Im\left(\psi_{s}(S, X)\right)}{2 \Im(\psi(S, X))} X_{\theta, \theta}+\frac{\Im\left(\psi_{s}(S, X)\right)}{2 \Im(\psi(S, X))} X_{\theta, x} .
\end{aligned}
$$

Using Cauchy-Riemann equations, we have

$$
\begin{aligned}
\Theta_{\theta, s} & =\frac{\Re\left(\phi_{s}\right)}{2 \Im(\phi)} \Theta_{\theta, \theta}+\frac{a^{\prime}}{a}\left(\frac{\Im\left(\psi_{s, s}(S, X)\right)}{2 \Im(\psi(S, X))}+\frac{\left(\Re\left(\psi_{s}(S, X)\right)\right)^{2}}{2(\Im(\psi(S, X)))^{2}}\right) X_{\theta}, \\
\Theta_{\theta, x}= & \left(\frac{\Re\left(\psi_{s, s}(S, X)\right)}{2 \Im(\psi(S, X))}-\frac{\Re\left(\psi_{s}(S, X)\right) \Im\left(\psi_{s}(S, X)\right)}{2(\Im(\psi(S, X)))^{2}}\right) X_{\theta} X_{x} \\
+ & \frac{\Im\left(\phi_{s}\right)}{2 \Im(\phi)}\left(\frac{\Re\left(\psi_{s, s}(S, X)\right)}{2 \Im(\psi(S, X))}-\frac{\Re\left(\psi_{s}(S, X)\right) \Im\left(\psi_{s}(S, X)\right)}{2(\Im(\psi(S, X)))}\right)\left(X_{\theta}\right)^{2} \\
& +\frac{\Im\left(\psi_{s}(S, X)\right)}{2 \Im(\psi(S, X))} X_{\theta, x}+\frac{\Im\left(\phi_{s}\right)}{2 \Im(\phi)} \frac{\Im\left(\psi_{s}(S, X)\right)}{2 \Im(\psi(S, X))} X_{\theta, \theta}-\frac{\Im\left(\phi_{s}\right)}{2 \Im(\phi)} \Theta_{\theta, \theta} .
\end{aligned}
$$


Moreover, differentiating (22) with respect to $x$ gives

$$
X_{x, s}=\left(\frac{\Re\left(\phi_{s, x}\right)}{2 \Im(\phi)}-\frac{\Re\left(\phi_{s}\right) \Im\left(\phi_{x}\right)}{2(\Im(\phi))^{2}}\right) X_{\theta}+\frac{\Re\left(\phi_{s}\right)}{2 \Im(\phi)} X_{x, \theta}
$$

and so,

$$
X_{x, s}=-\left(\frac{\Im\left(\phi_{s, s}\right)}{2 \Im(\phi)}+\frac{\left(\Re\left(\phi_{s}\right)\right)^{2}}{2(\Im(\phi))^{2}}\right) X_{\theta}+\frac{\Re\left(\phi_{s}\right)}{2 \Im(\phi)} X_{x, \theta}
$$

Then, differentiating (22) with respect to $\theta$ :

$$
X_{\theta, s}=\frac{\Re\left(\phi_{s}\right)}{2 \Im(\phi)} X_{\theta, \theta}
$$

Now, we differentiate (26) with respect to $x$

$$
\begin{aligned}
\Theta_{x, s}=\left(\frac{\Re\left(\phi_{s, x}\right)}{2 \Im(\phi)}-\frac{\Re\left(\phi_{s}\right) \Im\left(\phi_{x}\right)}{2(\Im(\phi))^{2}}\right) \Theta_{\theta}+\frac{\Re\left(\phi_{s}\right)}{2 \Im(\phi)} \Theta_{x, \theta} \\
\quad-\frac{a^{\prime}}{a}\left(\frac{\Re\left(\psi_{s, x}(S, X)\right)}{2 \Im(\psi(S, X))}-\frac{\Re\left(\psi_{s}(S, X)\right) \Im\left(\psi_{x}(S, X)\right)}{2(\Im(\psi(S, X)))^{2}}\right) X_{x}
\end{aligned}
$$

leading to:

$$
\begin{aligned}
\Theta_{x, s}=-\left(\frac{\Im\left(\phi_{s, s}\right)}{2 \Im(\phi)}+\frac{\left(\Re\left(\phi_{s}\right)\right)^{2}}{2(\Im(\phi))^{2}}\right) \Theta_{\theta}+\frac{\Re\left(\phi_{s}\right)}{2 \Im(\phi)} \Theta_{x, \theta} \\
+\frac{a^{\prime}}{a}\left(\frac{\Im\left(\psi_{s, s}(S, X)\right)}{2 \Im(\psi(S, X))}+\frac{\left(\Re\left(\psi_{s}(S, X)\right)\right)^{2}}{2(\Im(\psi(S, X)))^{2}}\right) X_{x}
\end{aligned}
$$

Differentiate (27) with respect to $s$ :

$$
\begin{aligned}
& \Theta_{s, x}= \frac{a^{\prime}}{a}\left(\frac{\Im\left(\psi_{s, s}(S, X)\right)}{2 \Im(\psi(S, X))}-\frac{\left(\Im\left(\psi_{s}(S, X)\right)\right)^{2}}{2\left(\Im(\psi(S, X))^{2}\right.}\right) X_{x} \\
&+\left(\frac{\Im\left(\psi_{s, x}(S, X)\right)}{2 \Im(\psi(S, X))}-\frac{\Im\left(\psi_{s}(S, X)\right) \Im\left(\psi_{x}(S, X)\right)}{2(\Im(\psi(S, X)))^{2}}\right) X_{s} X_{x}+\frac{\Im\left(\psi_{s}(S, X)\right)}{2 \Im(\psi(S, X))} X_{s, x} \\
& \quad+\frac{\Im\left(\psi_{s}(S, X)\right)}{2 \Im(\psi(S, X))}\left(\frac{\Im\left(\phi_{s, s}\right)}{2 \Im(\phi)}-\frac{\left(\Im\left(\phi_{s}\right)\right)^{2}}{2(\Im(\phi))^{2}}\right) X_{\theta} \\
&+\frac{\Im\left(\phi_{s}\right)}{2 \Im(\phi)}\left(\frac{\Im\left(\psi_{s, s}(S, X)\right)}{2 \Im(\psi(S, X))}-\frac{\left(\Im\left(\psi_{s}(S, X)\right)\right)^{2}}{2(\Im(\psi(S, X)))^{2}}\right) X_{\theta} \\
&+\frac{\Im\left(\phi_{s}\right)}{2 \Im(\phi)}\left(\frac{\Im\left(\psi_{s, x}(S, X)\right)}{2 \Im(\psi(S, X))}-\frac{\Im\left(\psi_{s}(S, X)\right) \Im\left(\psi_{x}(S, X)\right)}{2(\Im(\psi(S, X)))^{2}}\right) X_{s} X_{\theta} \\
&+\frac{\Im\left(\phi_{s}\right)}{2 \Im(\phi)} \frac{\left(\psi_{s}(S, X)\right)}{2 \Im(\psi(S, X))} X_{s, \theta}-\left(\frac{\Im\left(\phi_{s, s}\right)}{2 \Im(\phi)}-\frac{\left(\Im\left(\phi_{s}\right)\right)^{2}}{2(\Im(\phi))^{2}}\right) \Theta_{\theta}-\frac{\Im\left(\phi_{s}\right)}{2 \Im(\phi)} \Theta_{s, \theta}
\end{aligned}
$$


and replace $X_{s}$ by its value from (22), $X_{s, x}$ by (30), $X_{s, \theta}$ by (31), and $\Theta_{s, \theta}$ by (28):

$$
\begin{aligned}
\Theta_{s, x}= & \frac{a^{\prime}}{a}\left(\frac{\Im\left(\psi_{s, s}(S, X)\right)}{2 \Im(\psi(S, X))}-\frac{\left(\Im\left(\psi_{s}(S, X)\right)\right)^{2}}{2(\Im(\psi(S, X)))^{2}}\right) X_{x} \\
+ & \frac{\Re\left(\phi_{s}\right)}{2 \Im(\phi)}\left(\frac{\Re\left(\psi_{s, s}(S, X)\right)}{2 \Im(\psi(S, X))}-\frac{\Re\left(\psi_{s}(S, X)\right) \Im\left(\psi_{s}(S, X)\right)}{2(\Im(\psi(S, X)))^{2}}\right) X_{x} X_{\theta} \\
& -\frac{\Im\left(\psi_{s}(S, X)\right)}{2 \Im(\psi(S, X))} \frac{\left|\phi^{\prime}\right|^{2}}{2(\Im(\phi))^{2}} X_{\theta}+\frac{\Re\left(\phi_{s}\right)}{2 \Im(\phi)} \frac{\left.\psi_{s}(S, X)\right)}{2 \Im(\psi(S, X))} X_{x, \theta} \\
+ & \frac{a^{\prime}}{a} \frac{\Im\left(\phi_{s}\right)}{2 \Im(\phi)}\left(\frac{\Im\left(\psi_{s, s}(S, X)\right)}{2 \Im(\psi(S, X))}-\frac{\left(\Im\left(\psi_{s}(S, X)\right)\right)^{2}}{2(\Im(\psi(S, X)))^{2}}\right) X_{\theta} \\
+ & \frac{\Re\left(\phi_{s}\right) \Im\left(\phi_{s}\right)}{4(\Im(\phi))^{2}}\left(\frac{\Re\left(\psi_{s, s}(S, X)\right)}{2 \Im(\psi(S, X))}-\frac{\Re\left(\psi_{s}(S, X)\right) \Im\left(\psi_{s}(S, X)\right)}{2(\Im(\psi(S, X)))^{2}}\right)\left(X_{\theta}\right)^{2} \\
+ & \frac{\Re\left(\phi_{s}\right) \Im\left(\phi_{s}\right)}{4(\Im(\phi))^{2}} \frac{\Im\left(\psi_{s}(S, X)\right)}{2 \Im(\psi(S, X))} X_{\theta, \theta}-\left(\frac{\Im\left(\phi_{s, s}\right)}{2 \Im(\phi)}-\frac{\left(\Im\left(\phi_{s}\right)\right)^{2}}{2(\Im(\phi))^{2}}\right) \Theta_{\theta} \\
- & \frac{\Re\left(\phi_{s}\right) \Im\left(\phi_{s}\right)}{4(\Im(\phi))^{2}} \Theta_{\theta, \theta}-\frac{a^{\prime}}{a} \frac{\Im\left(\phi_{s}\right)}{2 \Im(\phi)}\left(\frac{\Im\left(\psi_{s, s}(S, X)\right)}{2 \Im(\psi(S, X))}+\frac{\left(\Re\left(\psi_{s}(S, X)\right)\right)^{2}}{2(\Im(\psi(S, X)))^{2}}\right) X_{\theta},
\end{aligned}
$$

where $\left|h^{\prime}\right|^{2}$ is the Jacobian determinant of $h$ for any holomorphic function. Now, since $\Theta_{s, x}=\Theta_{x, s}$ the previous two are equal. Thus, using the value of $\Theta_{x, \theta}$ found in (29), we get

$$
\Theta_{\theta}=\frac{a^{\prime}}{a} \frac{\left|\psi^{\prime}(S, X)\right|^{2}(\Im(\phi))^{2}}{\left|\phi^{\prime}\right|^{2}(\Im(\psi(S, X)))^{2}}\left(X_{x}+\frac{\Im\left(\phi_{s}\right)}{2 \Im(\phi)} X_{\theta}\right)+\frac{\Im\left(\psi_{s}(S, X)\right)}{2 \Im(\psi(S, X))} X_{\theta} .
$$

Replacing in (26) we have

$$
\begin{aligned}
\Theta_{s}=\frac{a^{\prime}}{a} \frac{\Re\left(\phi_{s}\right)}{2 \Im(\phi)} \frac{\left|\psi^{\prime}(S, X)\right|^{2}(\Im(\phi))^{2}}{\left|\phi^{\prime}\right|^{2}(\Im(\psi(S, X)))^{2}}\left(X_{x}+\frac{\Im\left(\phi_{s}\right)}{2 \Im(\phi)} X_{\theta}\right) \\
+\frac{\Re\left(\phi_{s}\right)}{2 \Im(\phi)} \frac{\Im\left(\psi_{s}(S, X)\right)}{2 \Im(\psi(S, X))} X_{\theta}-\frac{a^{\prime}}{a} \frac{\Re\left(\psi_{s}(S, X)\right)}{2 \Im(\psi(S, X))} .
\end{aligned}
$$

By Proposition 3.5, we can write

$$
\frac{\left|\psi^{\prime}(S, X)\right|^{2}(\Im(\phi))^{2}}{\left|\phi^{\prime}\right|^{2}(\Im(\psi(S, X)))^{2}}=h(., X)
$$

for a real valued function $h:] 0, b[\times] 0, b^{\prime}\left[\longrightarrow \mathbb{R}\right.$. We write $h_{2}$ the partial derivative of $h$ with respect to the second variable. Now, differentiate (34) with respect to $s$ :

$$
\begin{aligned}
\Theta_{s, \theta}=\frac{a^{\prime}}{a} h_{2}(., X) X_{s}\left(X_{x}+\frac{\Im\left(\phi_{s}\right)}{2 \Im(\phi)} X_{\theta}\right)+\frac{\Im\left(\psi_{s}(S, X)\right)}{2 \Im(\psi(S, X))} X_{s, \theta} \\
+\frac{a^{\prime}}{a} h(., X)\left(X_{s, x}+\left(\frac{\Im\left(\phi_{s, s}\right)}{2 \Im(\phi)}-\frac{\left(\Im\left(\phi_{s}\right)\right)^{2}}{2(\Im(\phi))^{2}}\right) X_{\theta}+\frac{\Im\left(\phi_{s}\right)}{2 \Im(\phi)} X_{s, \theta}\right) \\
+\frac{a^{\prime}}{a}\left(\frac{\Im\left(\psi_{s, s}(S, X)\right)}{2 \Im(\psi(S, X))}-\frac{\left(\Im\left(\psi_{s}(S, X)\right)\right)^{2}}{2(\Im(\psi(S, X)))^{2}}\right) X_{\theta} \\
+\left(\frac{\Im\left(\psi_{s, x}(S, X)\right)}{2 \Im(\psi(S, X))}-\frac{\Im\left(\psi_{s}(S, X)\right) \Im\left(\psi_{x}(S, X)\right)}{2(\Im(\psi(S, X)))^{2}}\right) X_{s} X_{\theta}
\end{aligned}
$$


and use formulas for $X_{s}$ and $X_{s, \theta}$ :

$$
\begin{aligned}
\Theta_{s, \theta} & =\frac{a^{\prime}}{a} h_{2}(., X) X_{\theta}\left(\frac{\Re\left(\phi_{s}\right)}{2 \Im(\phi)} X_{x}+\frac{\Re\left(\phi_{s}\right) \Im\left(\phi_{s}\right)}{4(\Im(\phi))^{2}} X_{\theta}\right) \\
& +\frac{a^{\prime}}{a} h(., X)\left(\frac{\Re\left(\phi_{s}\right)}{2 \Im(\phi)} X_{x, \theta}-\frac{\left|\phi^{\prime}\right|^{2}}{2(\Im(\phi))^{2}} X_{\theta}+\frac{\Re\left(\phi_{s}\right) \Im\left(\phi_{s}\right)}{4(\Im(\phi))^{2}} X_{\theta, \theta}\right) \\
& +\frac{\Re\left(\phi_{s}\right)}{2 \Im(\phi)}\left(\frac{\Re\left(\psi_{s, s}(S, X)\right)}{2 \Im(\psi(S, X))}+\frac{\Re\left(\psi_{s}(S, X)\right) \Im\left(\psi_{s}(S, X)\right)}{2(\Im(\psi(S, X)))^{2}}\right)\left(X_{\theta}\right)^{2} \\
+ & \frac{\Re\left(\phi_{s}\right)}{2 \Im(\phi)} \frac{\Im\left(\psi_{s}(S, X)\right)}{2 \Im(\psi(S, X))} X_{\theta, \theta}+\frac{a^{\prime}}{a}\left(\frac{\Im\left(\psi_{s, s}(S, X)\right)}{2 \Im(\psi(S, X))}-\frac{\left(\Im\left(\psi_{s}(S, X)\right)\right)^{2}}{2(\Im(\psi(S, X)))^{2}}\right) X_{\theta} .
\end{aligned}
$$

Now, differentiating (35) with respect to $\theta$ leads to:

$$
\begin{aligned}
\Theta_{\theta, s} & =\frac{a^{\prime}}{a} h_{2}(., X) X_{\theta}\left(\frac{\Re\left(\phi_{s}\right)}{2 \Im(\phi)} X_{x}+\frac{\Re\left(\phi_{s}\right) \Im\left(\phi_{s}\right)}{4(\Im(\phi))^{2}} X_{\theta}\right) \\
+ & \frac{\Re\left(\phi_{s}\right)}{2 \Im(\phi)}\left(\frac{\Re\left(\psi_{s, s}(S, X)\right)}{2 \Im(\psi(S, X))}-\frac{\Re\left(\psi_{s}(S, X)\right) \Im\left(\psi_{s}(S, X)\right)}{2(\Im(\psi(S, X)))^{2}}\right)\left(X_{\theta}\right)^{2} \\
+\frac{a^{\prime}}{a} \frac{\Re\left(\phi_{s}\right)}{2 \Im(\phi)} h(., X)\left(X_{x, \theta}+\right. & \left.\frac{\Im\left(\phi_{s}\right)}{2 \Im(\phi)} X_{\theta, \theta}\right)+\frac{\Re\left(\phi_{s}\right)}{2 \Im(\phi)} \frac{\Im\left(\psi_{s}(S, X)\right)}{2 \Im(\psi(S, X))} X_{\theta, \theta} \\
& +\frac{a^{\prime}}{a}\left(\frac{\Im\left(\psi_{s, s}(S, X)\right)}{2 \Im(\psi(S, X))}+\frac{\left(\Re\left(\psi_{s}(S, X)\right)\right)^{2}}{2(\Im(\psi(S, X)))^{2}}\right) X_{\theta} .
\end{aligned}
$$

Finally, using the fact that $\Theta_{s, \theta}=\Theta_{\theta, s}$ and the definition of $h$, we find

$$
\frac{\left|\psi^{\prime}(S, X)\right|^{2}}{(\Im(\psi(S, X)))^{2}} X_{\theta}=0
$$

which leads to

$$
X_{\theta}=0 \text {. }
$$

\section{ACKNOWLEDGMENTS}

The author would like to thank Ioannis Platis for enriching conversations regarding the subject of this article and the referee for a careful reading of the paper and for helpful comments.

\section{REFERENCES}

[Ahl66] Lars V. Ahlfors, Lectures on quasiconformal mappings, Manuscript prepared with the assistance of Clifford J. Earle, Jr. Van Nostrand Mathematical Studies, No. 10, D. Van Nostrand Co., Inc., Toronto, Ont.-New York-London, 1966. MR0200442

[Aus16] Alex D. Austin, Logarithmic Potentials and Quasiconformal Flows on the Heisenberg Group, ProQuest LLC, Ann Arbor, MI, 2016. Thesis (Ph.D.)-University of Illinois at Chicago. MR 3611265

[BFP13] Zoltán M. Balogh, Katrin Fässler, and Ioannis D. Platis, Modulus method and radial stretch map in the Heisenberg group, Ann. Acad. Sci. Fenn. Math. 38 (2013), no. 1, 149-180, DOI 10.5186/aasfm.2013.3811. MR3076803

[BFP15] Zoltán M. Balogh, Katrin Fässler, and Ioannis D. Platis, Uniqueness of minimisers for a Grötzsch-Belinskǐ type inequality in the Heisenberg group, Conform. Geom. Dyn. 19 (2015), 122-145, DOI 10.1090/ecgd/278. MR3343051

[BHIT06] Zoltán M. Balogh, Regula Hoefer-Isenegger, and Jeremy T. Tyson, Lifts of Lipschitz maps and horizontal fractals in the Heisenberg group, Ergodic Theory Dynam. Systems 26 (2006), no. 3, 621-651, DOI 10.1017/S0143385705000593. MR2237461 
[ACIDP16] A. Cano and I. D. Platis, Lifting of quasiconformal maps from the hyperbolic plane to the Heisenberg group (2016), available at https://www.researchgate.net/ project/Lifting-of-quasiconformal-maps-from-the-hyperbolic-plane-to-theHeisenberg-group.

[Cap97] Luca Capogna, Regularity of quasi-linear equations in the Heisenberg group, Comm. Pure Appl. Math. 50 (1997), no. 9, 867-889, DOI 10.1002/(SICI)10970312(199709)50:9<867::AID-CPA3〉3.0.CO;2-3. MR 1459590

[CT95] Luca Capogna and Puqi Tang, Uniform domains and quasiconformal mappings on the Heisenberg group, Manuscripta Math. 86 (1995), no. 3, 267-281, DOI 10.1007/BF02567994. MR1323792

[Kim14] Youngju Kim, Quasiconformal conjugacy classes of parabolic isometries of complex hyperbolic space, Pacific J. Math. 270 (2014), no. 1, 129-149, DOI 10.2140/pjm.2014.270.129. MR.3245851

[KR85] A. Korányi and H. M. Reimann, Quasiconformal mappings on the Heisenberg group, Invent. Math. 80 (1985), no. 2, 309-338, DOI 10.1007/BF01388609. MR788413

[KR95] A. Korányi and H. M. Reimann, Foundations for the theory of quasiconformal mappings on the Heisenberg group, Adv. Math. 111 (1995), no. 1, 1-87, DOI 10.1006/aima.1995.1017. MR.1317384

[KR87] Adam Korányi and Hans Martin Reimann, Horizontal normal vectors and conformal capacity of spherical rings in the Heisenberg group (English, with French summary), Bull. Sci. Math. (2) 111 (1987), no. 1, 3-21. MR886958

[Min94] Robert R. Miner, Quasiconformal equivalence of spherical CR manifolds, Ann. Acad. Sci. Fenn. Ser. A I Math. 19 (1994), no. 1, 83-93. MR1246889

[Tan96] Puqi Tang, Regularity and extremality of quasiconformal homeomorphisms on CR 3-manifolds, Ann. Acad. Sci. Fenn. Math. 21 (1996), no. 2, 289-308. MR 1404088

[Wan06] Wei Wang, The Teichmüller distance on the space of spherical CR structures, Sci. China Ser. A 49 (2006), no. 11, 1523-1538, DOI 10.1007/s11425-006-2052-y. $\operatorname{MR} 2288212$

Sorbonne Université, Université Paris Diderot, CNRS, Institut de Mathématiques de Jussieu-Paris Rive Gauche, IMJ-PRG, F-75005, Paris, France

Email address: robin.timsit@imj-prg.fr 